\title{
Tissue-specific metabolic reprogramming drives nutrient flux in diabetic complications
}

Kelli M. Sas, ${ }^{1}$ Pradeep Kayampilly, ${ }^{1}$ Jaeman Byun, ${ }^{1}$ Viji Nair, ${ }^{1,2}$ Lucy M. Hinder, ${ }^{3}$ Junguk Hur, ${ }^{4}$ Hongyu Zhang, ${ }^{1}$ Chengmao Lin, ${ }^{5}$ Nathan R. Qii, ${ }^{1}$ George Michailidis, ${ }^{6}$ Per-Henrik Groop, ${ }^{7,8,9}$ Robert G. Nelson, ${ }^{10}$ Manjula Darshi, ${ }^{11}$ Kumar Sharma, ${ }^{11}$ Jeffrey R. Schelling, ${ }^{12}$ John R. Sedor, ${ }^{12,13}$ Rodica Pop-Busui, ' Joel M. Weinberg, ' Scott A. Soleimanpour, ${ }^{1}$ Steven F. Abcouwer, ${ }^{5}$ Thomas W. Gardner, ${ }^{5}$ Charles F. Burant, ${ }^{1,14}$ Eva L. Feldman, ${ }^{3}$ Matthias Kretzler, ${ }^{1,2}$ Frank C. Brosius III, ${ }^{1,14}$ and Subramaniam Pennathur ${ }^{1}$

'Department of Internal Medicine, ${ }^{2}$ Department of Computational Medicine and Bioinformatics, ${ }^{3}$ Department of Neurology, University of Michigan, Ann Arbor, Michigan, USA. ${ }^{4}$ Department of Biomedical Sciences, University of North Dakota School of Medicine and Health Sciences, Grand Forks, North Dakota, USA. ${ }^{5}$ Department of Ophthalmology and Visual Sciences, ${ }^{6}$ Department of Statistics, University of Michigan, Ann Arbor, Michigan, USA. ${ }^{7}$ Folkhälsan Institute of Genetics, Folkhälsan Research Center, Biomedicum Helsinki, Helsinki, Finland. ${ }^{8}$ Abdominal Center Nephrology, University of Helsinki and Helsinki University Hospital, Helsinki, Finland. ${ }^{9}$ Baker IDI Heart and Diabetes Institute, Melbourne, Australia. ${ }^{10}$ Diabetes Epidemiology and Clinical Research Section, National Institute of Diabetes and Digestive and Kidney Diseases, National Institutes of Health, Phoenix, Arizona, USA. "Institute of Metabolomic Medicine and Center for Renal Translational Medicine, Department of Medicine, University of California San Diego, and Veterans Administration San Diego Healthcare System, La Jolla, California, USA. ${ }^{12}$ Department of Medicine, ${ }^{13}$ Department of Physiology and Biophysics, Case Western Reserve University, Cleveland, Ohio, USA. ${ }^{14}$ Department of Molecular and Integrative Physiology, University of Michigan, Ann Arbor, Michigan, USA.

Diabetes is associated with altered cellular metabolism, but how altered metabolism contributes to the development of diabetic complications is unknown. We used the BKS $d b / d b$ diabetic mouse model to investigate changes in carbohydrate and lipid metabolism in kidney cortex, peripheral nerve, and retina. A systems approach using transcriptomics, metabolomics, and metabolic flux analysis identified tissue-specific differences, with increased glucose and fatty acid metabolism in the kidney, a moderate increase in the retina, and a decrease in the nerve. In the kidney, increased metabolism was associated with enhanced protein acetylation and mitochondrial dysfunction. To confirm these findings in human disease, we analyzed diabetic kidney transcriptomic data and urinary metabolites from a cohort of Southwestern American Indians. The urinary findings were replicated in $\mathbf{2}$ independent patient cohorts, the Finnish Diabetic Nephropathy and the Family Investigation of Nephropathy and Diabetes studies. Increased concentrations of TCA cycle metabolites in urine, but not in plasma, predicted progression of diabetic kidney disease, and there was an enrichment of pathways involved in glycolysis and fatty acid and amino acid metabolism. Our findings highlight tissue-specific changes in metabolism in complication-prone tissues in diabetes and suggest that urinary TCA cycle intermediates are potential prognostic biomarkers of diabetic kidney disease progression.

Authorship note: K.M. Sas and P. Kayampilly contributed equally to this work.

Conflict of interest: $\mathrm{K}$. Sharma has an equity interest in ClinMet Inc.

Submitted: February 8, 2016 Accepted: August 16, 2016 Published: September 22, 2016

Reference information: JCI Insight. 2016;1(15):e86976. doi:10.1172/jici.insight.86976.

\section{Introduction}

Diabetes is characterized by altered carbohydrate and lipid metabolism. Despite medications that reduce hyperglycemia, hypertension, and hyperlipidemia, diabetes is often associated with highly morbid complications such as diabetic kidney disease (DKD), diabetic neuropathy (DN), and diabetic retinopathy (DR). Diabetes is the most common cause of end-stage renal disease, lower extremity amputation, and blindness in the United States (1). Despite the fact that most diabetic patients develop one or more of these complications, the pathophysiology leading to development and progression of these complications remains poorly understood. 
A

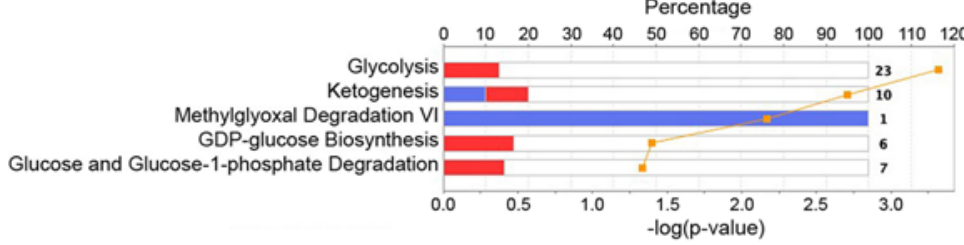

B

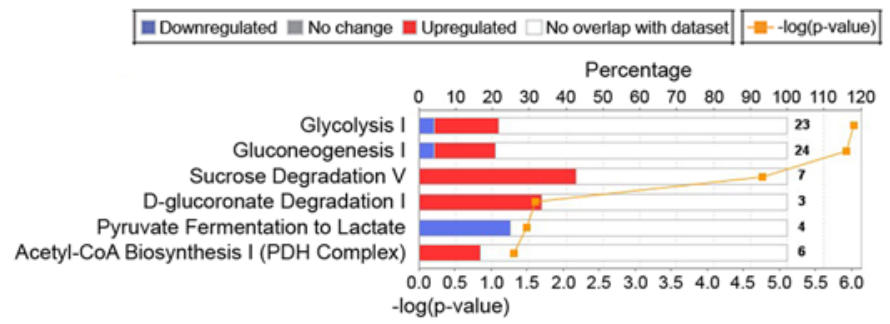

C

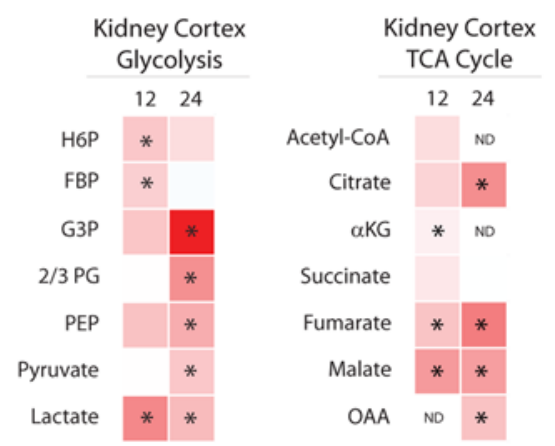

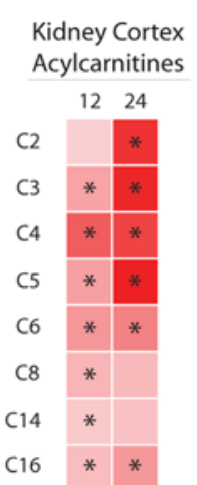

D
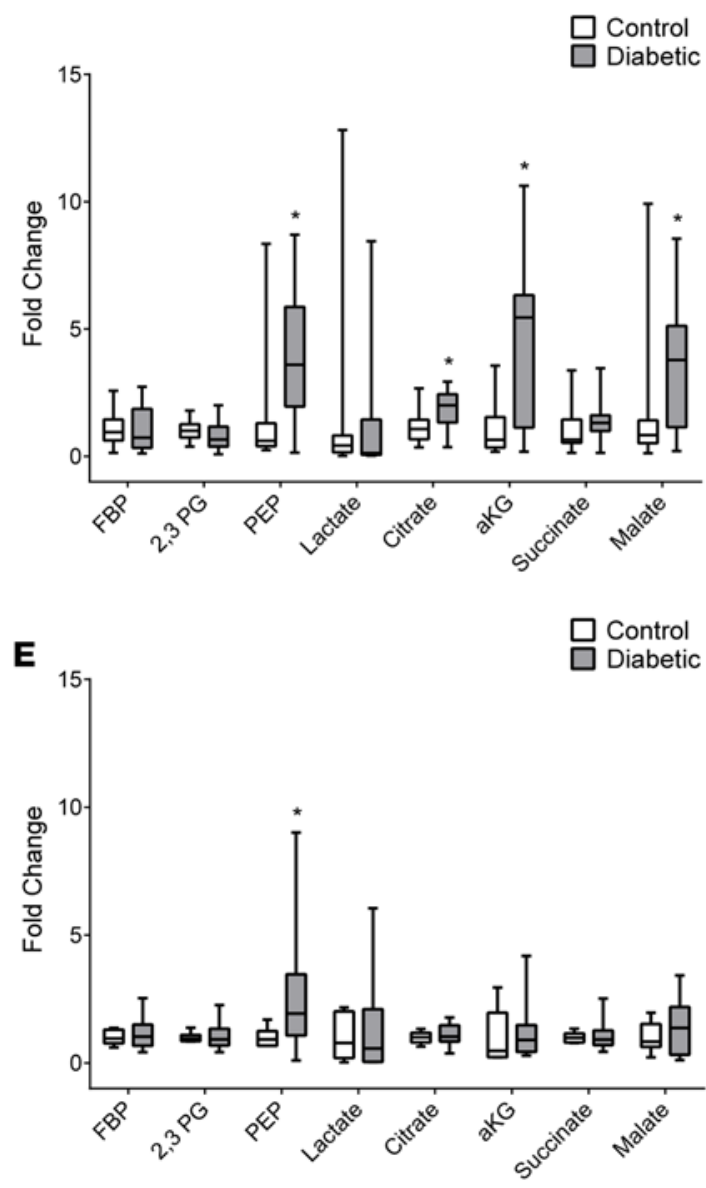

Figure 1. Transcriptomic and metabolomic analyses from control and diabetic mice. (A and B) Predicted alteration of pathways involved in glucose metabolism in the (A) kidney cortex and (B) sciatic nerve in 24-week-old diabetic versus control mice, with the percentage of genes upregulated (red) and downregulated (blue) $(P<0.05$ [- $\log (P$ value $)>1.3]$, kidney $n=5 /$ group; nerve control $n=9$, nerve diabetic $n=10)$. The number of transcripts in each pathway is shown at the right margin corresponding to each pathway. (C) Relative levels of glycolytic, TCA cycle, and acylcarnitine metabolites from kidney cortex of 12- and 24-week-old diabetic versus control mice are depicted as upregulated (red) or downregulated (blue) in diabetic mice ( $n=12 / \mathrm{group}$; ND, not detected above noise). ( $\mathbf{D}$ and $\mathbf{E}$ ) Levels of glycolytic and TCA cycle metabolites in urine from (D) 12- and (E) 24-week-old control and diabetic mice ( $n=5 /$ group). ${ }^{*} P<0.05$, Student's 2 -tailed $t$ test.

In type 1 and type 2 diabetes, increases in blood concentrations of glucose and non-esterified fatty acids are reflected intracellularly in the kidney, especially the proximal tubules (2-4). Though glomerular scarring is the hallmark pathological feature of early DKD and tubulointerstitial fibrosis is a later finding, proximal tubular dysfunction is now regarded as an early event in DKD pathogenesis (5). Although the proximal tubule is the primary site of glucose reabsorption from the glomerular filtrate, proximal tubule cells rely primarily on fatty acid oxidation to supply their high energy demand (6-8). Less is known about glucose and fatty acid metabolism in the peripheral nerve and retina (9-11). Because they are neuronal tissues, it is widely believed that both peripheral nerve and retina prefer glucose oxidation under normal conditions. The retina is unique in that it exhibits a high rate of aerobic glycolysis resembling the Warburg effect in cancer cells (12). Little is known about retinal lipid oxidation, although a recent study established the presence of fatty acid $\beta$-oxidation enzymes in Müller glial cells in the retina (13). Moreover, there is a lack of information on how metabolism in these two complication-prone tissues compares with kidney.

Mitochondrial fuel utilization is dependent upon both substrate availability and the relative ability to oxidize substrates. Given the increased plasma glucose and free fatty acid levels in poorly controlled diabetes, we sought to determine whether tissues vulnerable to diabetic complications alter their metabolism in response to changes in available nutrients. The main hypothesis of this study was that metabolic reprogramming, as evidenced by maladaptive changes in metabolism, would lead to tissue dysfunction. Identification 
Retina

Glycolysis

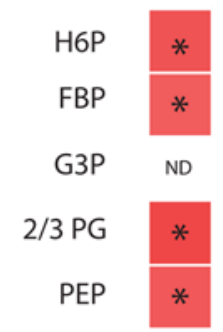

Pyruvate ND

Lactate ND
Retina

TCA Cycle

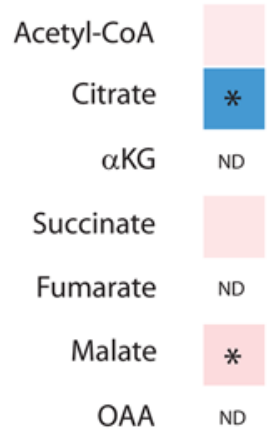

Retina

Acylcarnitines

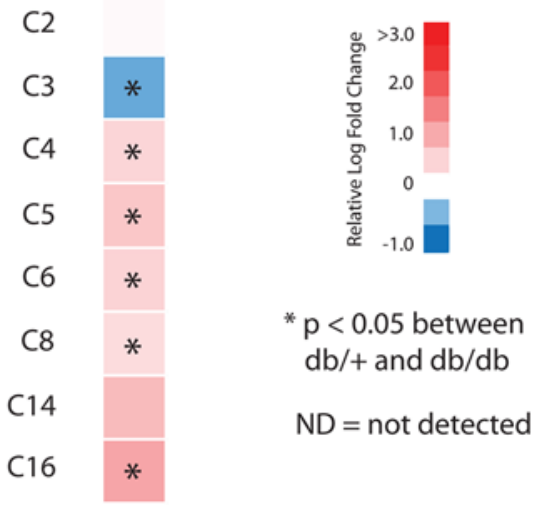

Figure 2. Metabolomics analysis of retina from control and diabetic mice. Relative levels of glycolytic, TCA cycle, and acylcarnitine metabolites from retina of 24-week-old diabetic versus control mice are depicted as upregulated (red) or downregulated (blue) in diabetic mice ( $n=10 /$ group; ND, not detected above noise). ${ }^{*} P<$ 0.05 , Student's 2 -tailed $t$ test.

of such alterations in metabolism could provide insights into the mechanisms of tissue damage, identify biomarkers, and uncover approaches to prevent or halt diabetic complications in individuals with diabetes. To obtain a comprehensive view of substrate metabolism, we used a systems-based approach combining transcriptomics and highly sensitive and specific mass spectrometry-based (MS-based) metabolomics and metabolic flux analyses to determine alterations in glucose and fatty acid metabolism. Transcriptomic and metabolomic profiling demonstrated an increase in glycolysis, fatty acid $\beta$-oxidation, and tricarboxylic acid (TCA) cycle flux in the diabetic kidney. These changes were tissue dependent, as metabolism in the nerve and retina was distinctive, with impaired glucose metabolism in the nerve and an increase in metabolism in the retina that was much less robust than that in the kidney. We found that increased excretion of TCA metabolites in human urine predicted DKD progression. The changes in kidney metabolism were associated with mitochondrial dysfunction and increased acetylation of several mitochondrial proteins, suggesting a link between altered glucose and fatty acid metabolism and kidney dysfunction early in DKD.

\section{Results}

Diabetes increases glycolysis, $\beta$-oxidation, and TCA cycle activity in the mouse kidney. Transcriptomic and metabolomic analyses were used to identify potential differences in intermediary metabolism in kidney cortex, predominantly comprising proximal tubule epithelia, from $d b / d b$ type 2 diabetic mice and $d b /+$ normoglycemic littermate controls (Figure 1). Targeted pathway analysis of the 24-week-old glomerular-depleted kidney cortex transcriptome using a list of 363 transcripts known to be involved in nutrient metabolism revealed significant enrichment primarily of pathways in fatty acid metabolism, with both $\beta$-oxidation and synthesis of more complex lipids (Supplemental Figure 1A; supplemental material available online with this article; doi:10.1172/jci.insight.86976DS1). Additionally, pathways involved in glucose and amino acid metabolism

Figure 3. Mitochondrial metabolism from kidney cortex of control and diabetic mice. Relative mitochondrial levels of TCA cycle and acylcarnitine metabolites from kidney cortex of 12- and 24-week-old diabetic versus control mice are depicted as upregulated (red) or downregulated (blue) in diabetic mice ( $n=10 /$ group; ND, not detected above noise). ${ }^{*} P<0.05$, Student's 2-tailed $t$ test.

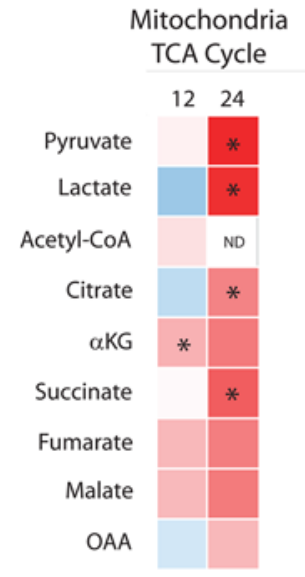


A

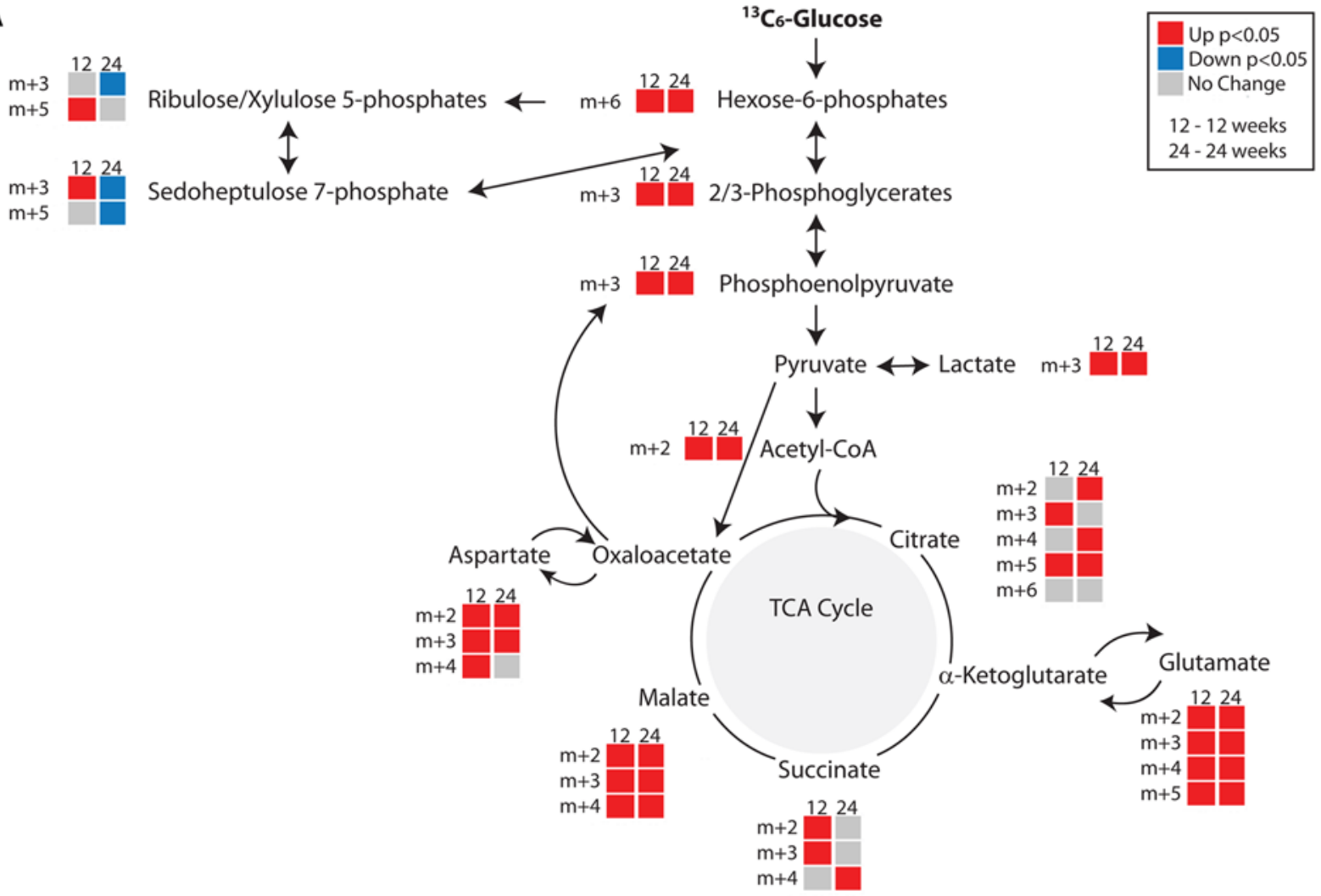

B
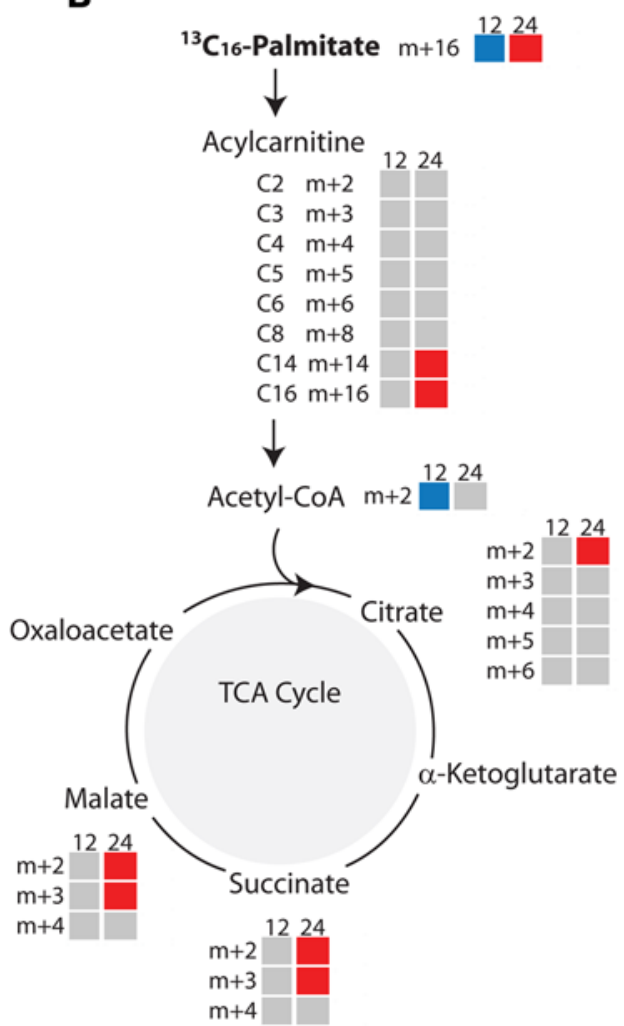

Up $p<0.05$

Down $p<0.05$

No Change

12 - 12 weeks

$24-24$ weeks
Figure 4. In vivo metabolic flux analyses in the kidney cortex of control and diabetic mice. Metabolic flux was determined from (A) $\left[{ }^{3} \mathrm{C}_{6}\right]$ glucose ( $n=8 /$ group) or (B) $\left[{ }^{13} C_{16}\right] \mathrm{K}$ palmitate (12 week, $n=6 /$ group; 24 week, control $n=5$, diabetic $n=8$ ). Metabolites in the diabetic kidney cortex from 12- and 24-week-old mice are depicted as upregulated (red), downregulated (blue), or unchanged (gray) compared with control kidney cortex. Upon entry into the TCA cycle through acetyl-CoA, each TCA cycle metabolite incorporates $2{ }^{13} \mathrm{C}$ labels $(m+2)$. Metabolites resulting from a second turn of the TCA cycle would incorporate 2 (citrate, $\mathrm{m}+4$ ) or 1 (all other intermediates, $\mathrm{m}+3$ ) additional ${ }^{13} \mathrm{C}$ labels. If labeled pyruvate enters the TCA cycle through oxaloacetate, it will contribute 3 or $5{ }^{13} \mathrm{C}$ labels $(m+3$ or $m+5)$ to citrate during condensation with unlabeled or labeled acetyl-CoA, respectively. ${ }^{*} P<0.05$, Student's 2-tailed $t$ test. 
were also significantly enriched (Figure 1A and Supplemental Figure 1B). There was significantly increased expression of mRNAs corresponding to glycolytic enzymes including hexokinase (Hk1), phosphofructokinase $(P f k l)$, and pyruvate kinase (Pklr), primary regulators of glycolytic flux (Supplemental Table 1). Transcripts for several enzymes involved in fatty acid metabolism were also significantly altered. The mRNA expression of 2 enzymes involved in $\beta$-oxidation were altered, with significantly increased expression of enoyl-CoA delta isomerase 1 (Ecil) and significantly decreased expression of enoyl-CoA-hydratase/3-hydroxyacyl-CoA dehydrogenase (Ehhadh) (Supplemental Table 1). We found no significant changes in expression of mRNAs for enzymes involved in the TCA cycle or enrichment of any TCA cycle pathways.

To determine whether the transcriptomic predictions resulted in functional alterations, we determined changes in metabolite levels in kidney cortex from 12- and 24-week-old diabetic and control mice, corresponding to early and established $\mathrm{DKD}$, respectively $(14,15)$. Kidney cortex was analyzed using targeted liquid chromatography-MS (LC/MS) and gas chromatography-MS (GC/MS) for $~ 65$ metabolites representing central carbon metabolism, including acyl-CoAs, acylcarnitines, and amino acids, as well as metabolites in glycolysis, the pentose phosphate pathway, and the TCA cycle (Figure 1C and Supplemental Table 2). Consistent with the transcriptomic data, several glycolytic metabolites were upregulated in the diabetic kidney, including the hexose 6-phosphates and pyruvate. Likewise, many TCA cycle intermediates were increased, including fumarate and malate. These changes were seen in both the 12- and 24-week-old diabetic mice. No significant changes were detected in pentose phosphate pathway metabolites or AMP/ ATP and ADP/ATP ratios in kidneys from 24-week-old mice (Supplemental Table 2).

Transcriptomic data also predicted increased fatty acid oxidation, and we found increases in both evenchain and odd-chain acylcarnitines in diabetic kidney cortex at 12 and 24 weeks (Figure 1C), without significant changes in long-chain acyl-CoAs (not shown). Acylcarnitines are important for shuttling fatty acids into the mitochondria for use as an energy source. Even-chain acylcarnitines are a metric of lipid $\beta$-oxidation, whereas odd-chain acylcarnitines (C3 and C5) are produced during the catabolism of branched-chain amino acids and can be used as anaplerotic substrates (16). Odd-chain acylcarnitines were increased in the diabetic kidney, suggesting increased branched-chain amino acid oxidation. Although there were no significant differences in the branched-chain amino acids themselves, these trended upward in the diabetic kidney while the majority of amino acid levels were decreased (Supplemental Table 2).

Concomitantly, we examined glycolytic and TCA cycle metabolites in mouse plasma and urine to assess whether these metabolite changes could be further examined as biomarkers. While no changes were detected in mouse plasma (not shown), both glycolytic (phosphoenolpyruvate) and TCA cycle (citrate, $\alpha$-ketoglutarate, malate) intermediates were increased in diabetic mouse urine (Figure 1, D and E), suggesting these could be markers of early DKD and might imply altered tubular metabolism or function.

Diabetes increases glycolysis, $\beta$-oxidation and TCA cycle enzyme transcripts in the mouse sciatic nerve, but decreases related metabolites. Transcriptomic and metabolomics analyses were used to identify potential differences in intermediary metabolism in $d b / d b$ sciatic nerve $(\mathrm{SCN})$ at 24 weeks of age (Figure $1 \mathrm{~B}$ and Supplemental Figure 2). This age corresponds to established DN pathology in the $d b / d b$ mouse model (17). Targeted pathway analysis of our previously published transcriptomic data (18) revealed significant enrichment of several pathways involved in fatty acid oxidation, glycolysis, catabolism of amino acids feeding into the TCA cycle and TCA cycle activity (Figure 1B and Supplemental Figure 2, A-C). Similar to findings in the kidney, we detected significantly increased expression of mRNAs encoding glycolytic enzymes, including hexokinase $3(H k 3)$, phosphofructokinase ( $P f k l$ ) and neuron-specific phosphopyruvate hydratase (Eno2) (18). Transcripts for several enzymes involved in fatty acid metabolism were also significantly upregulated, including the fatty acid transporter Slc27a1, long-chain acyl-CoA synthetase 1 (Acsl1), acyl-CoA oxidase 1 (Acox1), and hydroxyacyl-CoA dehydrogenase/3-ketoacyl-CoA thiolase/enoyl-CoA hydratase (Hadha) (18). The encoded enzymes are involved in insulin-stimulated fatty acid uptake, activation of long-chain fatty acids for metabolism, and the first and last three steps of $\beta$-oxidation, respectively. In contrast to the kidney, we observed significant changes in expression of mRNAs encoding TCA cycle enzymes, with increased expression of citrate synthase $(C s)$ and isocitrate dehydrogenase 3 (Idh3a), catalyzing the first and rate-limiting steps of the TCA cycle, respectively (18).

To assess whether the transcriptomic predictions resulted in functional alterations, we first determined changes in metabolite levels in SCN from 24-week-old control and diabetic mice. SCN was analyzed using targeted LC/MS for acyl-CoAs and acylcarnitines (Supplemental Figure 2D). Our previously published data on glycolytic and TCA cycle metabolites (10) were re-examined for comparative purposes 
A

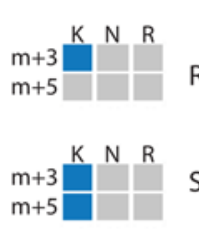

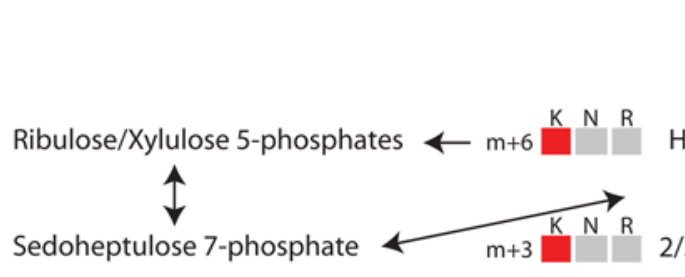

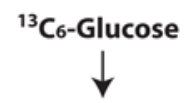

Hexose-6-phosphates

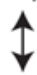

2/3-Phosphoglycerates

\begin{tabular}{|l|}
\hline Up $p<0.05$ \\
Down $p<0.05$ \\
No Change \\
K- Kidney \\
N- Nerve \\
R- Retina \\
\hline
\end{tabular}
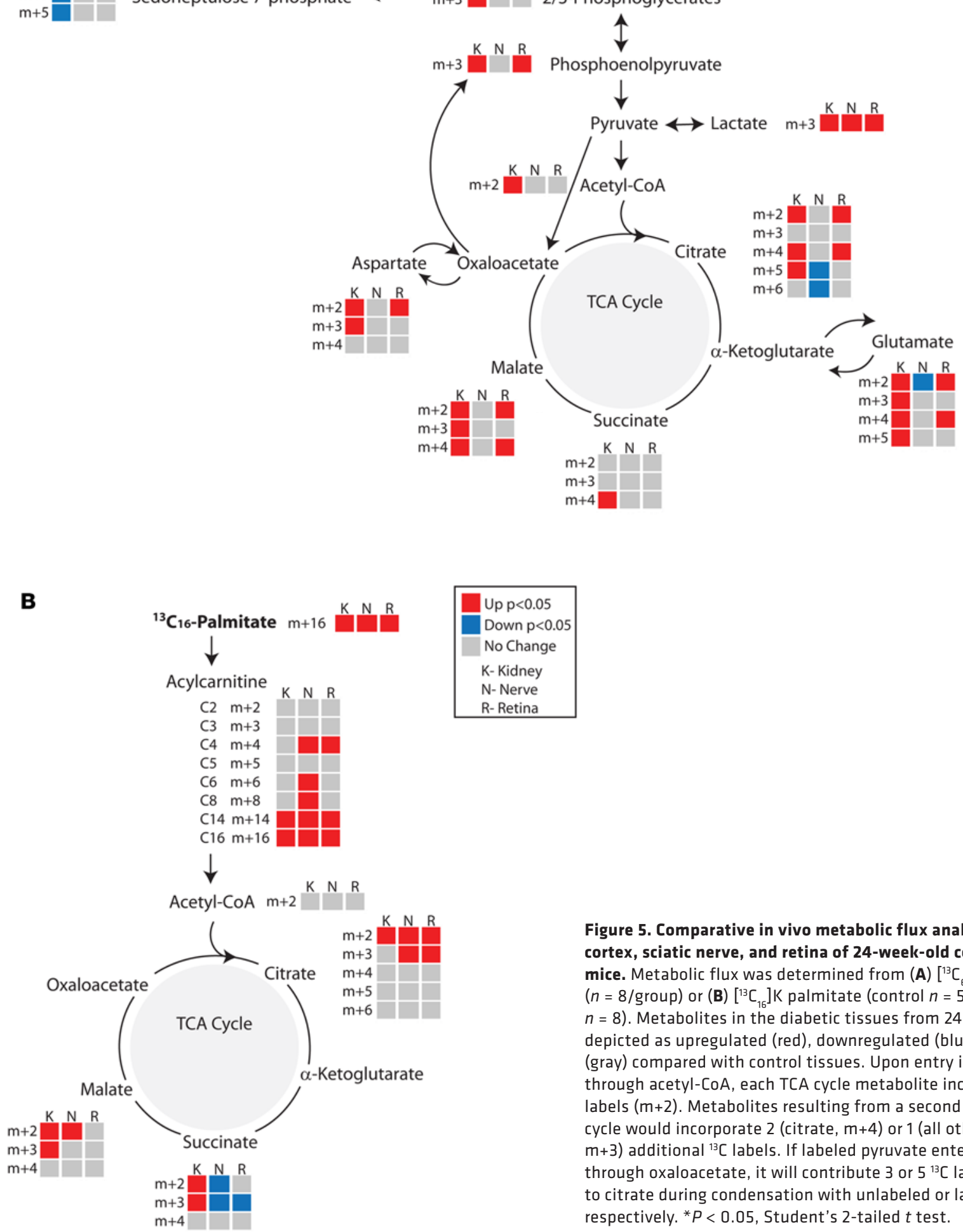

Up $p<0.05$

Down $\mathrm{p}<0.05$

No Change

K- Kidney

$\mathrm{N}$ - Nerve

R- Retina
Figure 5. Comparative in vivo metabolic flux analyses in the kidney cortex, sciatic nerve, and retina of 24-week-old control and diabetic mice. Metabolic flux was determined from (A) $\left[{ }^{13} C_{6}\right]$ glucose ( $n=8$ /group) or (B) $\left[{ }^{13} \mathrm{C}_{16}\right] \mathrm{K}$ palmitate (control $n=5$, diabetic $n=8$ ). Metabolites in the diabetic tissues from 24 -week-old mice are depicted as upregulated (red), downregulated (blue) or unchanged (gray) compared with control tissues. Upon entry into the TCA cycle through acetyl-CoA, each TCA cycle metabolite incorporates $2{ }^{13} \mathrm{C}$ labels $(m+2)$. Metabolites resulting from a second turn of the TCA cycle would incorporate 2 (citrate, $m+4$ ) or 1 (all other intermediates, $\mathrm{m}+3$ ) additional ${ }^{13} \mathrm{C}$ labels. If labeled pyruvate enters the TCA cycle through oxaloacetate, it will contribute 3 or $5^{13} \mathrm{C}$ labels $(m+3$ or $m+5)$ to citrate during condensation with unlabeled or labeled acetyl-CoA, respectively. ${ }^{*} P<0.05$, Student's 2 -tailed $t$ test. 
(Supplemental Figure 2D). In contrast to the transcriptomic data, glycolytic metabolites were downregulated in the diabetic SCN, including the hexose 6-phosphates, the phosphoglycerates, phosphoenolpyruvate, and lactate. Similarly, the TCA cycle intermediates citrate and isocitrate were decreased, even though mRNA expression of the involved enzymes was increased. Transcriptomic data also predicted increased fatty acid oxidation and, similar to the observations with glycolytic intermediates, we found decreases in both even- and odd-chain acylcarnitines along with long-chain acyl-CoAs in the diabetic SCN at 24 weeks (Supplemental Figure 2D). These data are consistent with an overall decrease in energy metabolism in diabetic SCN and demonstrate that different tissues exhibit different metabolic responses to the excess substrate available with diabetes. Additionally, these data highlight the importance of using a multiscalar systems biological approach as transcriptomic changes do not always predict changes in protein level and function or the ultimate metabolic phenotype.

Diabetes increases glycolysis and $\beta$-oxidation metabolites in the diabetic retina. Targeted metabolomics was used to identify potential differences in intermediary metabolism in retina from 24-week-old $d b / d b$ type 2 diabetic mice and $d b /+$ normoglycemic littermate controls (Figure 2). The $d b / d b$ mouse model at 16 or 24 weeks of age was recently identified as an appropriate model for mechanistic studies of diabetes-induced retinal neurodegeneration (19). Similar to the kidney but in contrast to peripheral nerve, glycolytic metabolite levels in the 24-week-old diabetic retina were significantly increased, along with long-chain acylcarnitines. Although metabolites reflecting proximal energy metabolism were increased, this pattern was not uniformly reflected by metabolites in the TCA cycle. There were no significant differences in acetyl-CoA, and although malate was significantly increased and succinate trended toward an increase $(P=0.0508)$, citrate was significantly decreased.

Mitochondrial metabolic alterations are reflective of tissue metabolism in the diabetic kidney. To determine whether the metabolite alterations in kidney cortex were reflected in mitochondria from this tissue, we prepared mitochondria and measured metabolite levels using LC/MS and GC/MS from the kidney cortex of 12- and 24-week-old control and diabetic mice (Figure 3 and Supplemental Table 3). Similar to whole kidney cortex, there were increases in several TCA cycle metabolites, including citrate and succinate, along with increased lactate, which can be oxidized in the mitochondria (20). No differences were detected in acyl-CoA levels (data not shown), but both even-chain and odd-chain acylcarnitines were increased. Along with the whole tissue data, these findings imply increased fatty acid catabolism in diabetic kidney cortex. The increased fatty acid catabolism did not appear to be due to incomplete oxidation, as the ratio of $\mathrm{C} 8$ to C16 acylcarnitine was not significantly different between groups (Supplemental Table 3). TCA cycle metabolites and acylcarnitines were significantly increased in the mitochondria from kidney cortex of 24-weekold compared with 12-week-old mice. This finding indicates a progressive increase in metabolism in the mitochondria later in DKD, suggesting either an increased need for metabolic energy production or an adaptation to consume excess metabolic fuel availability.

Diabetes progressively increases metabolic flux in the kidney. To confirm our interpretation of the static metabolite measurements, we used metabolic flux analysis to determine the rate of turnover of metabolites in glycolysis, fatty acid oxidation, the TCA cycle, and the pentose phosphate pathway in all 3 complication-prone tissues. Use of $\left[\mathrm{U}_{-}{ }^{13} \mathrm{C}_{6}\right]$ glucose or $\left[2,3-{ }^{13} \mathrm{C}_{2}\right]$ sodium pyruvate assessed metabolic flux through the glycolytic pathway and TCA cycle or flux through the TCA cycle independent of glycolysis, respectively. Metabolites were detected by LC/MS and GC/MS, and the percent isotopologue enrichment (percent ${ }^{13} \mathrm{C}$-labeled versus unlabeled) was determined.

Metabolic flux through glycolysis and the TCA cycle in the kidney of 12- and 24-week-old diabetic mice was increased compared with littermate controls (Figure 4A, Supplemental Figure 3A, and Supplemental Table 4). Using $\left[{ }^{13} \mathrm{C}_{6}\right]$ glucose, flux through both glycolysis and the TCA cycle was increased in the kidney cortex from both 12- and 24-week-old diabetic mice, while flux through the pentose phosphate pathway was decreased in the 24-week-old diabetic kidney (Figure 4A). Furthermore, carbon influx into the TCA cycle occurs through both acetyl-CoA and pyruvate. Isotope-labeled sodium pyruvate allowed for a closer examination of flux through the TCA cycle. The diabetic animals exhibited increased activity through the TCA cycle, even though there was no significant change in ATP levels (Supplemental Figure 3A and Supplemental Table 2). Although the relative abundance of glutamate and aspartate was lower or unaltered in 12- and 24-week-old diabetic kidney cortex (Supplemental Table 2), there was increased ${ }^{13} \mathrm{C}_{6}$ label incorporation, suggesting increased metabolic flux through these amino acids (Figure 4A and Supplemental Figure 3A). The decreased abundance along with the increased metabolism of glutamate 
A

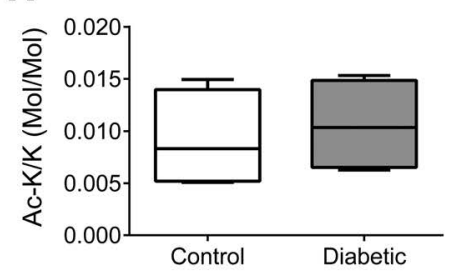

C
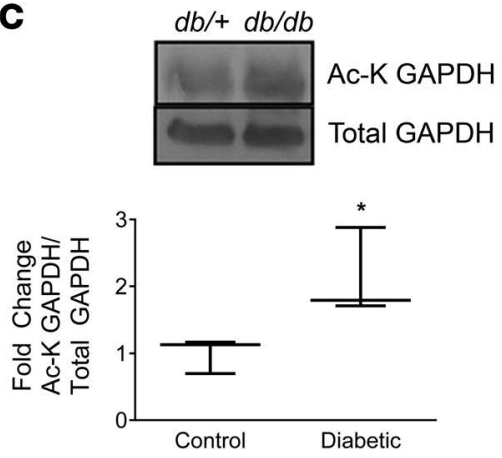

D
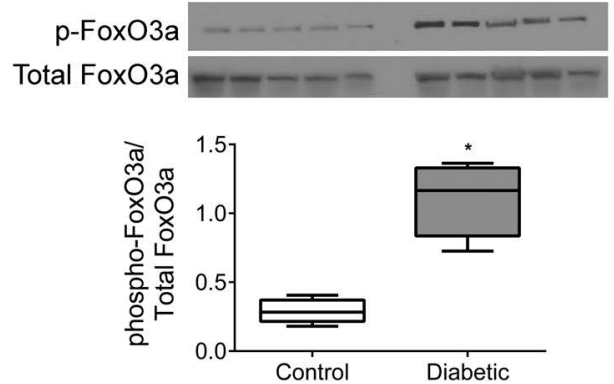

B

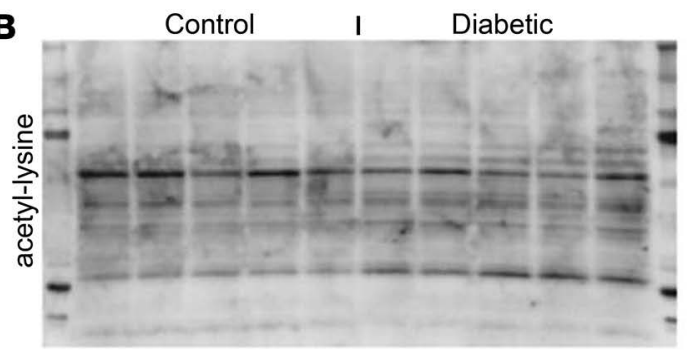

$d b /+d b / d b$
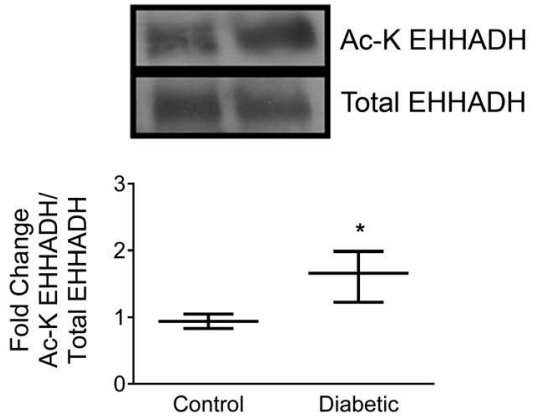

acetyl-FoxO3a

Total FoxO3a
Figure 6. Acetylation in the kidney cortex of 24-week-old control and diabetic mice. Total lysine acetylation was determined by (A) LC/MS and (B) Western blot of kidney cortex lysates from 24-week-old control ( $d b /+)$ and diabetic $(d b / d b)$ mice ( $n=5 /$ group). (c) Relative acetylation of GAPDH and enoyl-CoA-hydratase/3-hydroxyacyl-CoA dehydrogenase (EHHADH), enzymes involved in glycolysis and $\beta$-oxidation, respectively, were determined ( $n=3$ /group). (D) Relative phosphorylation and acetylation of Fox03a ( $n=3$ /group). ${ }^{*} P<0.05$, Student's 2-tailed $t$ test.

and aspartate and increased accumulation of odd-chain acylcarnitines are consistent with an increase in anaplerotic flux in the diabetic kidney.

We additionally examined metabolic flux through fatty acid metabolism using $\left[{ }^{13} \mathrm{C}_{16}\right]$ palmitate complexed to fatty acid-free BSA. Isotope incorporation into acylcarnitines and metabolites in the TCA cycle was determined and corrected for the percent of labeled palmitate $(m+16)$ in the blood. While fatty acid flux in the 12-week diabetic kidney cortex was not changed from controls, it was increased at 24 weeks in the diabetic animals (Figure 4B). Together these data suggest metabolic reprogramming during the course of diabetes, with a progressive increase in virtually all ATP-generating metabolic pathways by 24 weeks of age.

Metabolic flux in diabetes is tissue dependent. To compare changes in metabolic flux in other complication-prone tissues, we examined flux through glycolysis, the TCA cycle, and $\beta$-oxidation in the sciatic (peripheral) nerve and retina of 24-week-old diabetic and control mice and compared these findings with the diabetic kidney (Figure 5, Supplemental Figure 3B, and Supplemental Tables 4 and 5). Following $\left[\mathrm{U}_{-}{ }^{13} \mathrm{C}_{6}\right.$ ]glucose administration (Figure 5A), there was a significant decrease in TCA cycle flux in the diabetic nerve, although flux through most of glycolysis remained unchanged. Glucose metabolic flux through the retina was modestly increased, although not as strongly as in the kidney. Administration of $\left[2,3-{ }^{13} \mathrm{C}_{2}\right]$ sodium pyruvate resulted in increased flux through the TCA cycle in all 3 tissues (Supplemental Figure 3B). These results suggest a rate-limiting reduction in glucose uptake or glycolytic enzyme activity in the diabetic nerve, which could be bypassed by administration of pyruvate.

Fatty acid metabolic flux through $\beta$-oxidation was also tissue dependent. Following administration of $\left[\mathrm{U}_{-}{ }^{13} \mathrm{C}_{16}\right]$ palmitate, uptake into each of the 3 tissues was increased in diabetic animals (Figure $5 \mathrm{~B}$ ). However, while flux through the TCA cycle remained elevated in the diabetic kidney, flux through the 

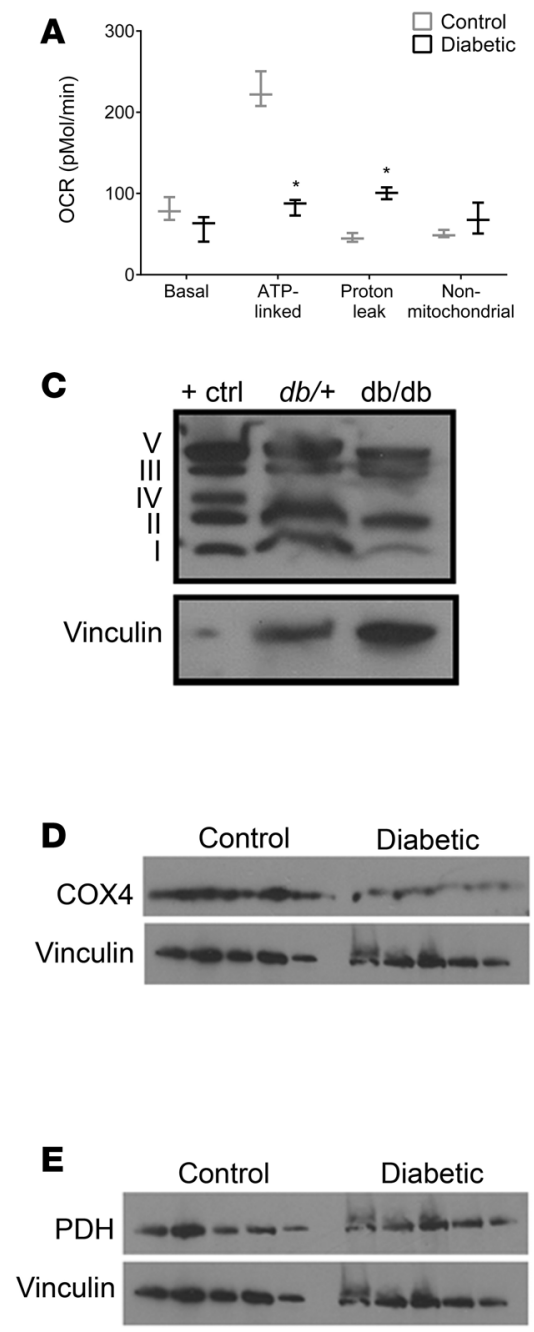
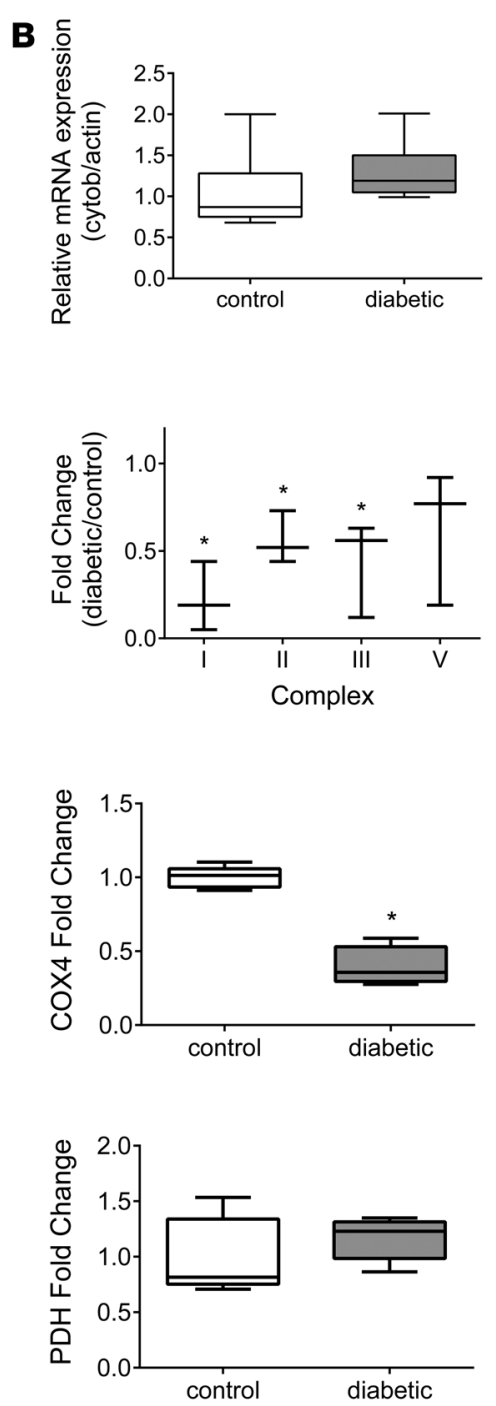

Figure 7. Mitochondrial electron transport chain complex function and expression in the kidney cortex of 24-week-old control and diabetic mice. (A) Oxygen consumption rate (OCR) was determined in mitochondria isolated from kidney cortex of 24-week-old control and diabetic mice to determine function through electron transport chain (ETC) complex I ( $n=3 /$ group). (B) Mitochondrial DNA copy number was determined by qPCR for cytochrome $b$ normalized to actin $(n=7 /$ group). Protein expression of (C) ETC complexes I, II, III, and V $(n=3)$, (D) cytochrome oxidase 4 (COX4), a subunit of complex IV ( $n=5$ /group), and (E) pyruvate dehydrogenase (PDH) $(n=$ 5/group) were normalized to vinculin. (D and E) The COX4 blot was reprobed for PDH; therefore, the same vinculin control is presented. ${ }^{*} P<0.05$, Student's 2-tailed $t$ test.

TCA cycle became diminished in the diabetic nerve and retina at succinate. Metabolism of succinate to fumarate produces $\mathrm{FADH}_{2}$, which is utilized for mitochondrial ATP synthesis directly via complex II of the electron transport chain. This suggests that although palmitate uptake was increased in both the nerve and retina, it was not fully utilized for energy metabolism. When taken together with diminished static levels of acylcarnitines and TCA cycle metabolites (Supplemental Figure 2D), these data point to either incomplete $\beta$-oxidation or preferential use of glucose-derived pyruvate as the major source for TCA cycle oxidation in peripheral nerve in diabetes.

Specific regulation of metabolic enzymes by acetylation. Acetylation is a nutrient-sensing post-translational modification, as its main substrate is acetyl-CoA (21), and is a major regulator of metabolic pathways $(22,23)$. To determine the effect of diabetes on total acetylation in mouse diabetic kidney cortex, we measured the amount of acetylated lysine residues by MS (Figure 6A) and analyzed protein acetylation by Western blotting (Figure 6B and Supplemental Figure 4A). Total acetylation was unchanged in kidney cortex of diabetic mice compared with controls, although the pattern of acetylated proteins was different. Therefore, we examined the acetylation of GAPDH and EHHADH, which are critical rate-limiting enzymes involved in glucose and fatty acid metabolism, respectively. Acetylation of both GAPDH and EHHADH (Figure 6C) was increased in the diabetic kidney. Acetylation of these enzymes enhances their activities (23,24). As global acetylation was not changed, the acetylation changes in specific metabolic enzymes were likely functionally important and not the result of a global response to increased substrate. Additionally, because diabetes caused increased $\beta$-oxidation as shown by metabolic flux studies (Figure 4) and gene expression of Ehhadh was actually decreased in the diabetic kidney, its acetylation appeared to play the dominant role in increasing its activity. We sought to examine the effects of increased nutrient availability and utilization on modification of forkhead box $\mathrm{O} 3$ (FoxO3), a transcription factor involved in the regulation of glucose and lipid metabolism. Phosphorylation and acetylation of FoxO3a were increased in the diabetic kidney (Figure $6 \mathrm{D}$ ), which would promote export from the nucleus and thereby impede transcription of target genes.

Increased metabolism is associated with mitochondrial dysfunction. Given the increase in glucose and fatty acid flux in the diabetic kidney cortex, we measured the oxygen consumption rate of mitochondria isolated from renal cortex of 24-week-old control and diabetic mice. Both glucose and fatty acids are major substrates for energy metabolism, with glycolysis providing NADH and $\beta$-oxidation providing both $\mathrm{NADH}$ and $\mathrm{FADH}_{2}$ for complexes I and II of the electron transport chain, respectively. Despite our prediction of increased oxidative phosphorylation and an increase in ATP production, we found evidence instead for enhanced proton 
A

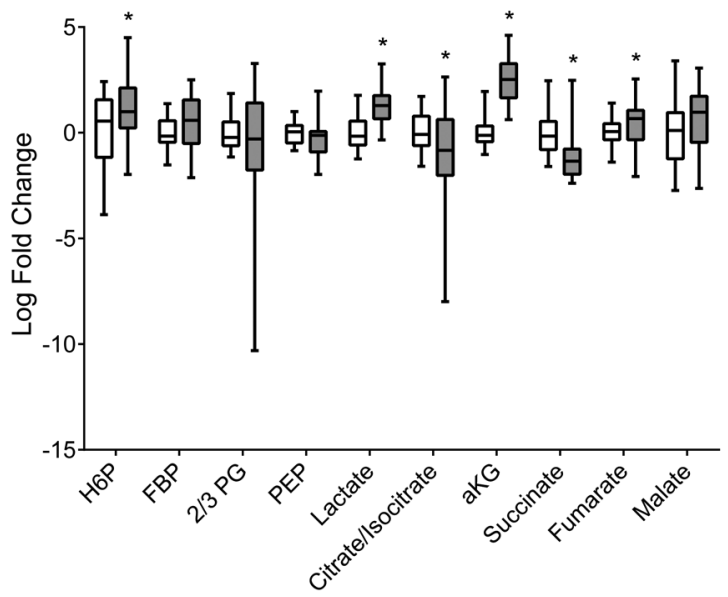

C

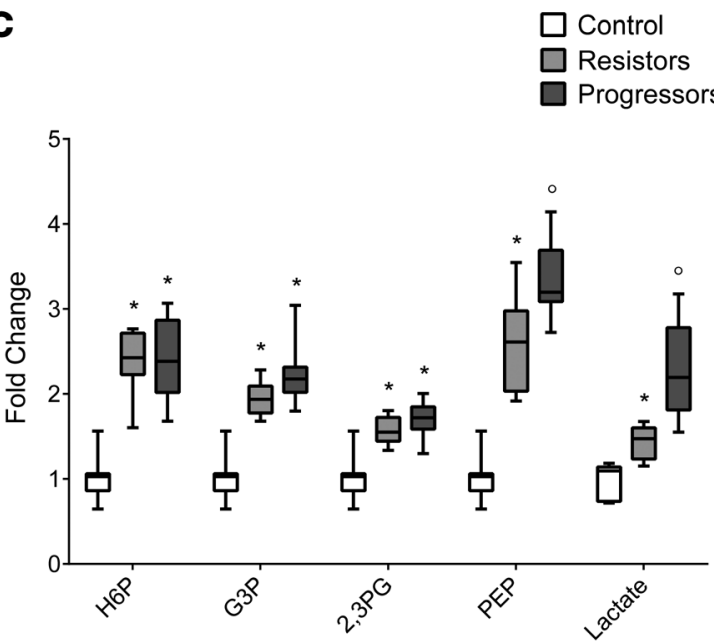

B

$\square$ Control

$\square$ Diabetic

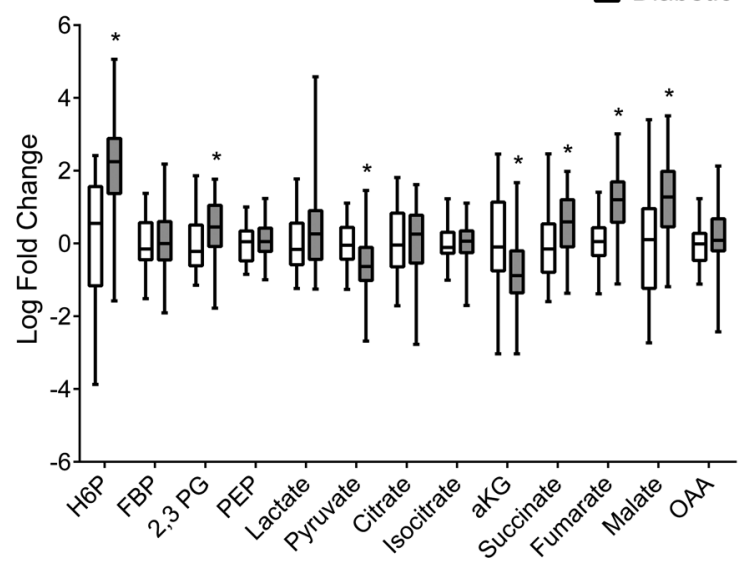

D
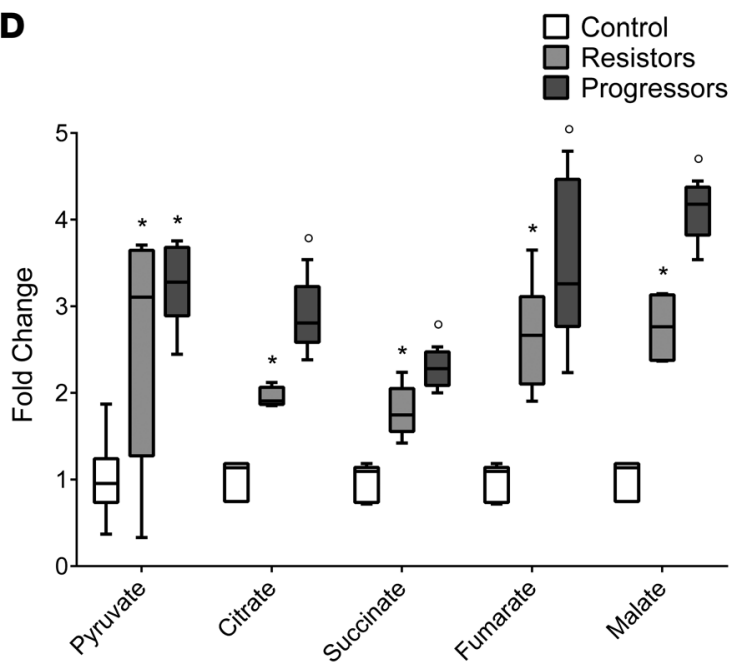

Figure 8. Metabolomic analysis of urines from diabetic and control subjects. (A and B) Levels of glycolytic and TCA cycle metabolites in urine from diabetic subjects from the (A) Southwestern American Indian cohort $(n=26)$ or (B) FinnDiane study $(n=72)$ compared with control subjects ( $n=28)$ enrolled in the FinnDiane study. ${ }^{*} P<0.05$, Student's 2 -tailed $t$ test. (C and $\left.\mathbf{D}\right)$ Levels of (C) glycolytic and (D) TCA cycle metabolites in baseline urine from control, diabetic resistor, and diabetic progressor subjects enrolled in the FIND study ( $n=10 /$ group). ${ }^{*} P \leq 0.05$ versus controls; ${ }^{\circ} P \leq 0.05$ versus controls and resistors, 1-way ANOVA with Tukey's multiple comparisons.

leak and diminished mitochondrial ATP production, as ADP-stimulated (state 3) respiration was lower following interrogation of both complex I (Figure 7A) and complex II (data not shown) in isolated mitochondria from diabetic mouse kidneys. Additionally, FCCP-uncoupled respiration, a marker for total mitochondrial capacity (25), was decreased in diabetic mitochondria, and expression of mitochondrial uncoupling protein 2 was increased 4-fold (Supplemental Figure 4B). The results of these studies suggest that electron transport chain dysfunction, leading to less efficient ATP production and compensatory increases in glucose and fatty acid metabolic flux, may be the primary metabolic abnormality in the diabetic kidney cortex.

The majority of ATP in aerobic metabolism is generated through the electron transport chain. Because ATP production-linked respiration was suppressed in the diabetic kidney, we examined the expression of the mitochondrial electron transport chain complexes by Western blotting. In renal cortex from diabetic mice, expression of proteins in complexes I, II, and III were significantly decreased at 24 weeks (Figure 7C) though not at 12 weeks (data not shown). Protein expression of complex IV subunit cytochrome $c$ oxidase subunit 4 (COX4) was also significantly decreased in mitochondria from 24-week diabetic kidneys (Figure 7D). There was no difference in mitochondrial DNA copy number between the control and diabetic mice (Figure 7B). Therefore, the reduction in complex I-IV expression was not due to an overall decrease in the number of mitochondria. To determine whether the decreased expression of electron 
Table 1. Clinical characteristics of Southwestern American Indian and FinnDiane study participants

\begin{tabular}{|c|c|c|c|}
\hline & Control subjects & Type 1 diabetic subjects & Type 2 diabetic subjects \\
\hline Age (yr) & $47.0(35.8,57.8)$ & $55.0(48.0,61.0)^{\mathrm{A}}$ & $44.7(37.2,52.4)$ \\
\hline European descent & $15(53.6 \%)$ & $72(100 \%)^{A}$ & $0(0 \%)$ \\
\hline Hispanic & $3(10.7 \%)$ & $0(0 \%)$ & $0(0 \%)$ \\
\hline Asian & $10(35.7 \%)$ & $0(0 \%)$ & $0(0 \%)$ \\
\hline Male & $20(71.4 \%)$ & $41(56.9 \%)$ & $8(30.8 \%)^{A}$ \\
\hline Female & $8(28.6 \%)$ & $31(43.1 \%)$ & $18(69.2 \%)^{A}$ \\
\hline Diabetes duration (yr) & N/A & $19.5(10.0,30.8)$ & $13.4(11.1,15.7)$ \\
\hline HbA1c (\%) & $5.5(5.4,6.0)(n=16)$ & $8.1(7.6,8.8)^{A}$ & $9.2(8.3,10.9)^{A}$ \\
\hline eGFR (ml/min/1.73 $\left.\mathrm{m}^{2}\right)$ & $104.0(86.0,134.8)(n=16)$ & $90.0(70.3,105.3)^{A}$ & \\
\hline \multicolumn{4}{|l|}{ Medication history (\%) ${ }^{\mathrm{B}}$} \\
\hline ACEi & $1(6.3 \%)$ & $24(33.3 \%)^{A}$ & $9(34.6 \%)^{A}$ \\
\hline ARB & $0(0 \%)$ & $10(13.9 \%)^{A}$ & $2(7.7 \%)^{A}$ \\
\hline
\end{tabular}

Values given as median (25\%, $75 \%$ ). Control and type 1 diabetic subjects enrolled in FinnDiane study and type 2 diabetic subjects from the Southwestern American Indians study. HbA1c, hemoglobin A1c; eGFR, estimated glomerular filtration rate; iGFR, iothalamate glomerular filtration rate; ACR, albumin to creatinine ratio; SBP, systolic blood pressure; DBP, diastolic blood pressure; ACEi, angiotensin-converting enzyme inhibitor; ARB, angiotensin receptor blocker. ${ }^{A} P<0.05$ versus control, $\chi^{2}$ test (ethnicity, medication history), Fisher exact test (age, sex), or 2 -tailed Student's $t$ test. ${ }^{B}$ Medication history from examination date closest to collection in type 2 diabetic subjects.

transport chain complexes was transcriptionally regulated, we examined our kidney cortex transcriptomic data for the expression of 84 nuclear-encoded individual subunits of the electron transport chain complexes. The expression of only one gene (Cox6b2), in complex IV, was significantly altered, with its expression being significantly increased in diabetic mice compared with controls (data not shown). No significant differences were detected in genes encoding complex I, II, III, or V subunits. No significant differences were detected in the gene expression levels of cytochrome $c$ oxidase subunit 4 (Cox4), although its protein expression was decreased (Figure 7D). To examine whether decreased protein expression was restricted to the electron transport chain proteins or reflected a more general reduction in expression of mitochondrial proteins, we measured pyruvate dehydrogenase $(\mathrm{PDH})$ levels by Western blotting (Figure 7E). No differences were detected in mRNA levels (Supplemental Table 1) or protein expression of PDH, suggesting that the changes in electron transport chain complex protein levels were relatively specific.

TCA cycle intermediates are biomarkers of progression to DKD in humans. To determine whether the transcriptional and metabolic changes found in the diabetic mice were recapitulated in humans with diabetes (26), we performed transcriptomic analysis of kidney biopsy samples obtained from 49 well-characterized Southwestern American Indians with type 2 diabetes $(27,28)$. Similar to the mouse data, targeted pathway analysis identified significant enrichment of many pathways involved in fatty acid/lipid metabolism, along with several in glucose and amino acid metabolism (Supplemental Figure 5, A-D). However, unlike in the mouse, targeted pathway analysis also showed increased expression in TCA cycle and associated metabolic cofactor pathways in the humans (Supplemental Figure 5D). Urine collected at time of biopsy in 26 subjects with hyperfiltration and microalbuminuria was analyzed to determine levels of glycolytic and TCA cycle metabolites (Table 1 and Figure 8A). Urine metabolite levels were compared with healthy controls (Table 1). The metabolite changes were mapped to the human transcriptome. The kidney transcriptome and urine metabolome were concordant (Supplemental Figure 6), and the network analysis was similar to that in the $d b / d b$ mice (Supplemental Figure 7). This analysis suggests that changes in urinary metabolites may reflect kidney metabolism and supports the use of urinary metabolites as potential biomarkers in kidney disease. 
Table 2. Clinical characteristics of FIND study subjects at baseline

\begin{tabular}{|c|c|c|c|}
\hline & Controls & Resistors & Progressors \\
\hline Number of subjects & 10 & 10 & 10 \\
\hline Age (yr) & $50(48.5,51.3)$ & $52(42.8,65)$ & $67(52.8,74.5)$ \\
\hline European descent & & $4(40 \%)$ & $4(40 \%)$ \\
\hline African American & & $6(60 \%)$ & $6(60 \%)$ \\
\hline \multicolumn{4}{|l|}{ Sex } \\
\hline Diabetes duration (yr) & $\mathrm{N} / \mathrm{A}$ & $22.0(13.3,26.3)$ & $17.5(11.5,23.5)$ \\
\hline HbA1c (\%) & & $7.1(6.9,8.6)$ & $7.7(6.6,8.2)$ \\
\hline Creatinine (mg/dl), baseline & $0.84(0.81,1.00)$ & $0.80(0.70,0.93)$ & $0.80(0.70,1.00)$ \\
\hline Creatinine (mg/dl), final & & $0.71(0.59,0.80)$ & $1.49(1.38,1.98)^{A}$ \\
\hline eGFR $\left(\mathrm{ml} / \mathrm{min} / 1.73 \mathrm{~m}^{2}\right)$, baseline & $91.5(73.8,103.3)$ & $91.8(76.9,102.8)$ & $93.3(71.7,103.9)$ \\
\hline Mean DBP (mmHg) & & $74.0(71.7,81.4)$ & $82.0(78.5,84.7)$ \\
\hline \multicolumn{4}{|l|}{ Medication history (\%) } \\
\hline ACEi & & $3(30 \%)$ & $4(40 \%)$ \\
\hline ARB & & $2(20 \%)$ & $4(40 \%)$ \\
\hline
\end{tabular}

Values given as median (25\%, 75\%). HbA1c, hemoglobin A1c; eGFR, estimated glomerular filtration rate; SBP, systolic blood pressure; DBP, diastolic blood pressure; $A C E i$, angiotensin-converting enzyme inhibitor; ARB, angiotensin receptor blocker. ${ }^{A} P<0.05$ between resistors and progressors; 2 -tailed Student's $t$ test, Welch's unequal variances $t$ test, 1-way ANOVA with Tukey's multiple comparisons, or Kruskal-Wallis with Dunn's multiple comparisons.

To examine whether these changes in metabolite levels were also evident in persons with type 1 diabetes, urine was obtained from a cohort of type 1 diabetic subjects with microalbuminuria and decreased estimated glomerular filtration rate (eGFR) and matched healthy control human subjects enrolled in the Finnish Diabetic Nephropathy (FinnDiane) Study (29) (Table 1). Increased glycolytic (hexose-6-phosphates, 2,3-phosphoglycerate) and TCA cycle metabolites (succinate, fumarate, malate) were detected in the urine of type 1 diabetic compared with healthy control subjects, while 2 intermediates (pyruvate, $\alpha$-ketoglutarate) were decreased in type 1 diabetic samples (Figure 8B). Urine acylcarnitine levels were similar in diabetic and nondiabetic urine samples (data not shown). Subgroup analysis of diabetic study participants on an angiotensin-converting enzyme inhibitor (ACEi) or angiotensin receptor blocker (ARB) $(n=34)$ and those that were not $(n=38)$ identified changes in urinary metabolite levels, although the direction of change was not uniform (Supplemental Figure 8). For example, urinary fumarate levels were higher from patients taking ACEi/ARB, while citrate, isocitrate, and oxaloacetate were lower. There were no significant differences in age or duration of diabetes between these groups, and there was a small but nonsignificant change in eGFR (mean 82.0 versus $90.9, P=0.2865$ ) and urine albumin to creatinine ratio (median $40.7 \mathrm{mg} / \mathrm{g}$ versus $6.4 \mathrm{mg} / \mathrm{g}, P=0.0661$ by Kruskal-Wallis test with Dunn's multiple comparisons).

To pursue the use of urinary metabolites as biomarkers of DKD progression, we further investigated acylcarnitines and glycolytic and TCA cycle intermediates in urine from a subset of patients from the Family Investigation of Nephropathy and Diabetes (FIND) study (30). Baseline samples were analyzed from non-diabetic control subjects and type 2 diabetic subjects who had no clinical evidence of DKD. A subset of diabetic subjects developed progressive DKD (progressors) defined by eGFR $<65 \mathrm{ml} / \mathrm{min} / 1.73 \mathrm{~m}^{2}$ and creatinine $>1.2 \mathrm{mg} / \mathrm{dl}$, and a subset of diabetic subjects did not develop DKD (resistors) at mean follow-up of 5 years (Table 2). Baseline $\mathrm{HbA}_{1 \mathrm{c}}$ levels were equivalent in both diabetic groups. Additionally, there were no significant differences in the degree of microalbuminuria at baseline between progressors and resistors. In the baseline fasting plasma samples, there were no significant differences in acylcarnitines or glycolytic or TCA cycle intermediates between the groups (data not shown). However, glycolytic intermediates were increased in the baseline urine samples of diabetic subjects compared with control subjects (Figure 8C), 


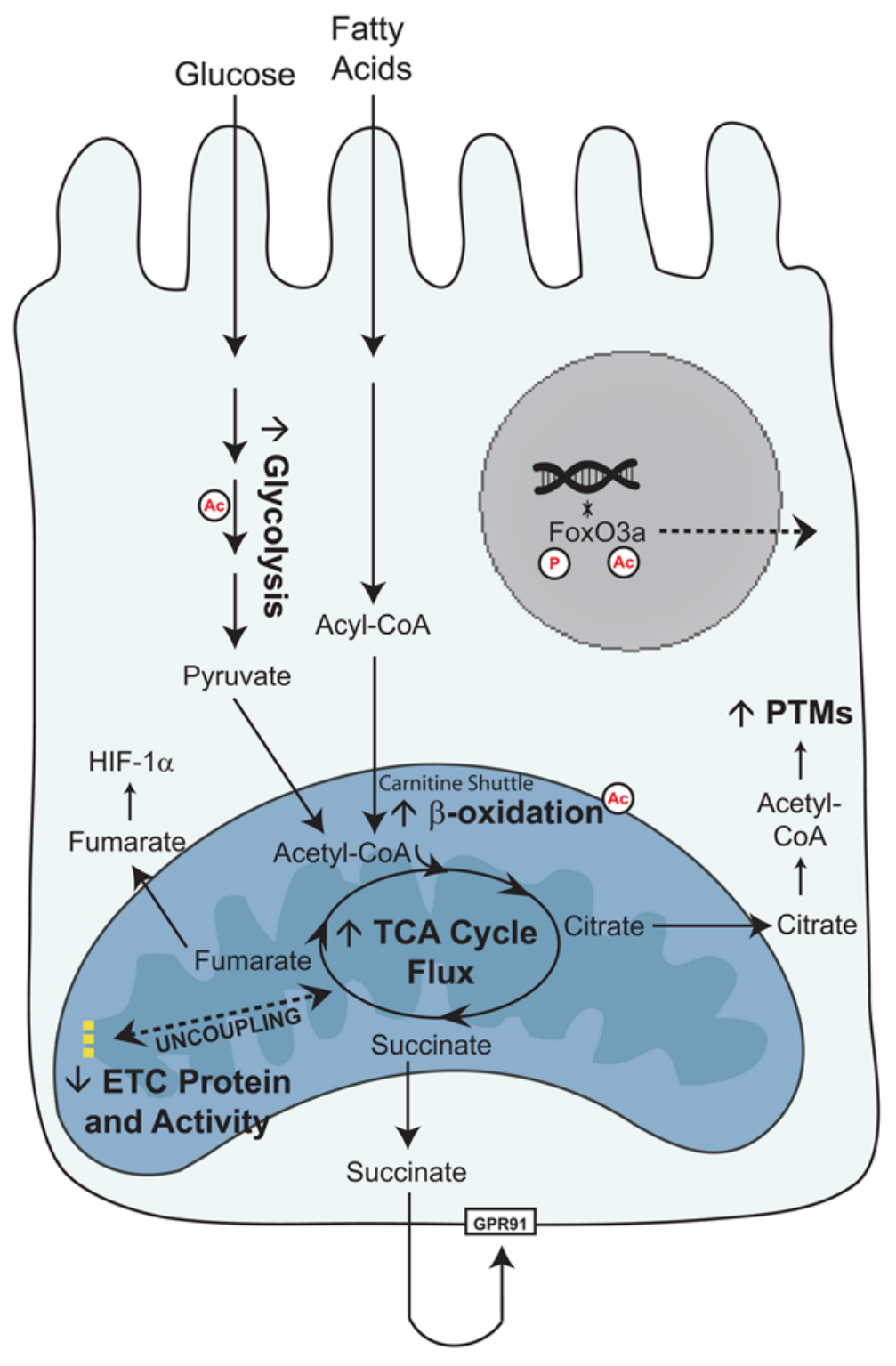

Figure 9. Schema of glucose and fatty acid metabolism in the diabetic proximal tubule cell. As the glucose concentration in plasma or urine increases, more glucose is transported into kidney proximal tubule cells. Glycolysis increases, resulting in increased pyruvate levels and TCA cycle activity in the mitochondria. Fatty acids are broken down into acyl-CoAs and transported across the mitochondrial membrane as acylcarnitines. $\beta$-Oxidation increases, resulting in increased TCA cycle activity. Although mitochondrial metabolism is elevated, there is a concurrent lack of increased ATP production through the electron transport chain (26). We propose that an uncoupling between mitochondrial metabolism and oxidative phosphorylation may underlie the metabolic phenotype of diabetic kidney disease. The increased TCA cycle metabolites can perturb normal cellular function. High levels of citrate can be used as a substrate for post-translational modifications (PTMs), such as acetylation (Ac), which can alter the activity of metabolic enzymes and localization of transcription factors. Succinate, through GPR91, can activate the renin-angiotensin system, and fumarate can induce HIF- $1 \alpha$. These perturbations can promote progression of DKD.

although most did not differentiate between progressors and resistors. Like the glycolytic metabolites, TCA cycle intermediates were elevated in diabetic subjects. Unlike the glycolytic metabolites, levels of most of the TCA cycle metabolites in baseline urine samples were significantly higher (1.3- to 1.6-fold) in progressors than in resistors (Figure 8D). The elevated metabolites in progressors decreased at final time of follow-up with disease progression (Supplemental Figure 9). This finding suggests that TCA cycle intermediates in urine could be used as a potential biomarker of the future development of progressive DKD.

\section{Discussion}

The prevailing theory of how hyperglycemia results in complications is through the glucose-derived increase in mitochondrial superoxide production $(31,32)$. The original experiments, conducted in an endothelial cell line, suggested a unifying mechanism for hyperglycemia-induced damage that resulted in the overproduction of reactive oxygen species, increased flux through the pentose phosphate pathway and decreased flux through the TCA cycle due to impaired GAPDH function $(33,34)$. A recent article challenged this theory, finding reduced superoxide production in the diabetic kidney along with reduced mitochondrial function, evidenced by decreased respiration (35). It was hypothesized that reduced mitochondrial biogenesis may have contributed over time to the switch from anaplerosis to cataplerosis. Here, we present data that also place mitochondrial dysfunction in a central role in DKD but suggest that there is an uncoupling between mitochondrial metabolism and oxidative phosphorylation.

The Randle hypothesis, or the glucose-fatty acid cycle, postulates that there is competition between glucose and fatty acids for their oxidation (36). The kidney cortex is $\sim 90 \%$ proximal tubules and favors $\beta$-oxidation but is exposed to increased levels of glucose during periods of hyperglycemia (6). Due to the increased intracellular glucose concentrations, it is not surprising that increased glycolytic and TCA cycle metabolites were found in the diabetic kidney cortex. However, we also found evidence of increased $\beta$-oxidation of fatty acids in the diabetic kidney. Our findings suggest that in the diabetic kidney there is a progressive increase in substrate utilization over time, with glucose and fatty acids being consumed simultaneously. These findings were predicted by mouse cortex transcriptomic analyses, as mRNA expression of enzymes involved in both glycolysis and fatty acid oxidation were increased (Figure 1A and Supple- 
mental Figure 1). The use of a systems approach identified a unique and heretofore unreported shift in the diabetic kidney toward substrate-driven metabolic adaptation.

Peripheral nerve and retina are other tissues severely affected by diabetes. Unlike kidney proximal tubules, peripheral nerves rely heavily on glucose for metabolism (9). Recently, the diabetic peripheral nerve was found to have diminished levels of glycolytic and TCA cycle metabolites, showing that not all complication-prone organs experience the same metabolic consequence of diabetes (10). The idea of tissue-specific pathological alterations in energy metabolism led us to investigate metabolic flux through the diabetic kidney, nerve, and retina. We found that metabolic flux in response to hyperglycemia is different and distinct in the 3 complication-prone tissues. Diabetic peripheral nerve showed a decrease in glucose oxidation and an increase in fatty acid oxidation. The metabolomics data were not predicted by peripheral nerve transcriptomic analysis, which suggested increased use of both glucose and fatty acids. This highlights the importance of using a multiscalar systems approach as the transcriptome does not always accurately predict the phenome. The discordance between the transcriptome, static metabolite levels and metabolic flux data suggests that other factors, such as post-transcriptional modifications, post-translational modifications, or changes in mitochondrial dynamics, are important in metabolic alterations in the diabetic nerve and the development of DN. The diabetic retina was more similar to the diabetic kidney in that oxidation of both glucose and fatty acid was increased, although more modestly than in the kidney, and fatty acid oxidation was incomplete, as reduced flux into succinate would result in limited production of $\mathrm{FADH}_{2}$ for ATP synthesis. Therefore, instead of a unifying metabolic mechanism for diabetic complications, it is more likely that complications arise in the context of distinct tissue-specific alterations in metabolism. Although DKD, DN, and DR are commonly referred to as "microvascular" complications, our results argue against this nomenclature, as the metabolite changes were not only tissue specific, but detected in the tissue parenchyma itself and not the microvasculature.

We propose that metabolic reprogramming and mitochondrial dysfunction may be at the root of diabetic complications, at least in the kidney (Figure 9). Surprisingly, we found increased central carbon substrate metabolism to be associated with diminished ATP-linked oxygen consumption in the diabetic kidney. Our data consistently identify mitochondrial alterations, but it remains unclear whether increased flux through the TCA cycle is causative or reactive to mitochondrial dysfunction. There are several ways in which mitochondrial dysfunction could result in increased TCA cycle flux. Accumulation of reactive oxygen species at the mitochondrial electron transport chain could result in damage to, or degradation of, the electron transport chain complexes (37). Indeed, in this study we found reduced protein expression of the mitochondrial electron transport chain complexes with very few corresponding changes in mRNA expression, suggesting that mRNA translation is diminished or that protein degradation is accelerated. Furthermore, these changes appear to be specific to the electron transport chain complexes, as not all mitochondrial proteins were altered. However, superoxide levels were recently found to be decreased in the diabetic kidney, and increasing superoxide production was protective (35). Uncoupling of the respiratory chain can prevent superoxide production, which would result in diminished ATP production (38). This would be congruent with the findings of reduced superoxide production in DKD (35), and we found an increase in uncoupling protein 2 in the diabetic mitochondria (Supplemental Figure 4B).

There are several ways in which metabolic reprogramming could result in specific alterations of mitochondrial function and promote DKD progression. First, increased generation of metabolic intermediates may be toxic. Succinate, a ligand of the G protein-coupled receptor GPR91, can activate the renin-angiotensin system (39-41), and the activation of angiotensin II may result in NADPH oxidase 4-generated (Nox4-generated) mitochondrial oxidative damage $(42,43)$, leading to a feed-forward mechanism that promotes kidney injury in diabetes. Fumarate, which also accumulates in the diabetic kidney and urine of a type 1 model of DKD, is a potent inducer of HIF- $1 \alpha$ and TGF- $\beta$, both of which could contribute to kidney disease pathology (44). Second, metabolic intermediates may alter metabolic enzyme activity through the generation of post-translational modifications. Citrate, a key intermediate in the mitochondrial TCA cycle, can be shuttled to the cytosol and converted by ATP citrate lyase to acetyl-CoA, the primary substrate for acetylating proteins to modify function. Recently, diabetes has been shown to result in acetylation of enzymes primarily regulating glycolysis, the TCA cycle, and branchedchain amino acid metabolism (45) and in acetylation of histones, which may lead to metabolic memory (46). Citrate was increased in our model, along with the acetylation of several metabolic enzymes. 
Although metabolic flux through the TCA cycle was increased, expression of mRNAs corresponding to TCA cycle enzymes was not significantly different in the diabetic kidney (Supplemental Table 1), supporting a role for post-translational modifications impacting mitochondrial function. Additionally, we found increased acetylation and phosphorylation of the transcription factor FoxO3. Post-translational modifications can alter transcription factor activity by promoting retention or export from the nucleus. The FoxO transcriptional factors regulate diverse cellular functions, including glucose and lipid metabolism $(47,48)$, and help protect against oxidative stress $(49,50)$. FoxO3a, the most abundant kidney isoform, is sensitive to energy and nutrient levels $(51,52)$, and caloric restriction has been shown to induce deacetylation (53). Although future work is needed to assess the relationship between acetylation/phosphorylation of FoxO3 and mitochondrial function in kidney disease, phosphorylation of FoxO3 is elevated in other animal models of progressive kidney disease (54) and fibrotic kidney disease (55). Additionally, phosphorylation of FoxO1 was elevated in the kidney of a type 1 diabetic mouse model and was associated with enhanced oxidative stress and fibrosis (56). Third, a defect in mitochondrial turnover could be perpetuated by increased metabolic flux. Previous studies have suggested that mitophagy is impaired in the diabetic kidney (57), and mitophagy is linked to mitochondrial bioenergetics $(58,59)$.

In support of the idea of metabolic reprogramming resulting in downstream complications, we found that glycolytic and TCA cycle metabolite levels in baseline human urine, but not plasma, separated subjects with early DKD from controls and, more importantly, predicted progression to DKD. TCA cycle metabolites in the urine could be useful prognostic biomarkers for DKD progression if these initial findings are corroborated in larger studies in different cohorts. As these changes were prominent in urine collected before progression of $\mathrm{DKD}$, the findings also suggest that these metabolite changes may be mechanistically important in DKD pathogenesis. As TCA cycle metabolites were elevated in the diabetic resistors over controls, although to a lesser extent than the diabetic progressors, these patients may continue on to develop DKD, albeit at a slower rate than the early progressors. Further studies are needed to determine whether elevation of steady-state urinary TCA cycle metabolites results from acute glycosuria or intrinsic differences in kidney metabolism or tubular handling. In vivo metabolic flux analysis following euglycemic and hyperglycemic clamp studies (in healthy controls and patients with and without established DKD) using isotopically enriched glucose to measure the production of core glucose-derived metabolites in blood and urine will help ascertain the underlying mechanisms.

The results from patients with early DKD differ from those with advanced DKD (eGFR 35 ml/ $\min / 1.73 \mathrm{~m}^{2}$ ), in whom decreased levels of TCA cycle metabolites were detected (60). Gene expression data also fit this pattern, as the expression of several genes involved in lipid metabolism was decreased in advanced DKD (61) and was strongly correlated with GFR, suggesting expression may decline with kidney function (62). It is likely that samples from advanced DKD subjects reflected the extensive tubular damage that is characteristic of that stage of the disease process, whereas those in early DKD patients reflect the increased metabolic activity of proximal tubular cells. A recent report examining TCA cycle metabolites over time in the serum and urine of $d b / d b$ diabetic and control mice supports this idea (63). Our finding that TCA cycle metabolites trended downward at final time of follow-up in progressors in the FIND study also lends support to this concept (Supplemental Figure 9). Interestingly, subgroup analysis on urinary metabolite levels based on renin-angiotensin-aldosterone system (RAAS) inhibitor intake revealed an increase in urinary fumarate but a decrease in urinary citrate, isocitrate, and oxaloacetate (Supplemental Figure 8). This implies that factors beyond filtration such as RAAS inhibition could also affect metabolite excretion. Further larger cohort studies are needed to confirm and validate prognostic capability of the TCA cycle metabolites in DKD progression. Future studies are also needed to determine the independent effects of renal filtration and RAAS inhibition on urinary metabolite levels. Regardless, changes in metabolism throughout the disease process highlight metabolic reprogramming as an important mechanism in the progression of DKD.

In conclusion, we used the pathophysiologically relevant BKS $d b / d b$ mouse model of type 2 diabetes mellitus to identify increased metabolic flux along with mitochondrial dysfunction in the diabetic kidney, which appear to reflect important processes in early human DKD and to be predictors of future progression of the disease. Similar to basal differences between kidney, nerve, and retina metabolism, the metabolic response to elevated glucose and fatty acids is unique in these 3 tissues. The findings reported here provide insight into the early features of $\mathrm{DKD}, \mathrm{DN}$, and $\mathrm{DR}$ and demonstrate that diabetes results in metabolic reprogramming in a tissue-specific manner in kidney cortex, peripheral nerve, and retina. 


\section{Methods}

For complete information on techniques used, refer to the Supplemental Methods.

Animal studies. Male BKS $d b / d b$ mice (BKS.Cg-m +/+ Lepr $\left.{ }^{\mathrm{db}} / \mathrm{J}\right)$ and littermate controls $(d b /+)$ were used at 12 and 24 weeks of age, corresponding to very early and established, but still early, DKD, respectively $(14,15)$. Prior to sacrifice and following a 2-hour fast, blood and urine were collected. Upon sacrifice, blood in the kidneys was removed by perfusion with ice-cold PBS. Kidneys were collected, and renal cortex was dissected. Samples were snap frozen and stored at $-80^{\circ} \mathrm{C}$ until use.

Metabolic flux analysis studies were performed by the Animal Phenotyping Core at the University of Michigan. All mice were fasted 2 hours prior to initiation of experiments. For metabolic flux analysis with isotope-labeled glucose and pyruvate, diabetic mice received $200 \mathrm{mU} / \mathrm{kg}$ insulin by i.p. injection. Control and diabetic mice received either $2 \mathrm{~g} / \mathrm{kg}\left[\mathrm{U}_{-}{ }^{13} \mathrm{C}_{6}\right]$ glucose or $2 \mathrm{~g} / \mathrm{kg}\left[{ }^{12} \mathrm{C}\right]$ glucose $+2 \mathrm{~g} / \mathrm{kg}\left[2,3-{ }^{13} \mathrm{C}_{2}\right]$ sodium pyruvate. Blood was collected via tail vein at $0,15,30,60,90,120$, and 150 minutes. For metabolic flux analysis with isotope-labeled palmitate, control and diabetic mice received $0.5 \mathrm{~g} / \mathrm{kg}\left[\mathrm{U}_{-}{ }^{13} \mathrm{C}_{16}\right]$ potassium palmitate complexed 6:1 with fatty acid-free BSA by gavage. Blood was collected via tail vein at $0,30,60$, 90, 120, 180, and 240 minutes. All animals were euthanized by cervical dislocation. Following sacrifice, all animals were perfused with ice-cold PBS, and kidney cortex, SCN, and retina were harvested, snap frozen, and stored at $-80^{\circ} \mathrm{C}$ until use.

Human samples. For transcriptomic analysis, kidney biopsy specimens were procured from 49 Southwestern American Indians enrolled in a randomized, placebo-controlled clinical trial to evaluate the renoprotective efficacy of losartan in type 2 diabetes (ClinicalTrials.gov NCT00340678) (28). GFR was measured by the urinary clearance of iothalamate as previously described (64). Subjects had a median GFR of $156.5 \mathrm{ml} / \mathrm{min}$ (range $81.0-267.0 \mathrm{ml} / \mathrm{min}$ ) and median urinary albumin/creatinine ratio (ACR) of 27.3 $\mathrm{mg} / \mathrm{g}$ (range $0.1-384.6 \mathrm{mg} / \mathrm{g}$ ). Sample processing, microdissection, and RNA isolation from microdissected tubulointerstitium were performed as previously described $(65,66)$. The fragmentation, hybridization, staining, and imaging were performed according to the Affymetrix Expression Analysis Technical Manual (Affymetrix). Affymetrix HGU133A and HGU133 Plus 2.0 Arrays were used in this study. The CEL files were normalized using the Robust MultiArray method and annotated with the Human Entrez Genes custom cdf version 10 (http://brainarray.mbni.med.umich.edu), as implemented in the GenePattern pipeline (http://genepattern.broadinstitute.org). Normalized expression value data were $\log _{2}$ transformed and batch corrected. For analysis of changes in kidney tubule gene expression, samples were compared with biopsies from 32 non-diabetic healthy living donors with no albuminuria. All processing followed procedures similar to those above. Significance Analysis of Microarrays in MeV TiGR Software (http://www. tm4.org/mev.html) was used to compute the fold change differences in genes. Significance was assessed at a false discovery rate (FDR) of $<0.1$. Downstream functional analysis of enriched pathways was generated using Ingenuity Pathway Analysis (QIAGEN). Owing to ethical considerations and privacy protection and to avoid identifying study participants, the Ethics Board of the National Institute of Diabetes and Digestive and Kidney Diseases has stipulated that individual-level gene expression and genotype data from this study cannot be made publicly available.

The FinnDiane and FIND study protocols, patient recruitment procedures, and patient classification criteria have been previously reported $(30,67)$. Patients in the FIND study were part of the ancillary Diabetic Kidney Disease Progression Study. Targeted analysis of plasma and urine samples by LC/MS was performed as described in Supplemental Methods.

Mouse transcriptomics. Total RNA were extracted from 24-week-old mouse SCN samples (control $n=9$, diabetic $n=10$ ) and analyzed as previously described (18). Total RNA were extracted from 24-week-old mouse kidney cortex samples ( $n=5$ /group) using the RNeasy Mini Kit (QIAGEN). Gene expression profiling was performed using Affymetrix Mouse Genome 4302.0 arrays according to the manufacturer's instructions. The raw image files (CEL files) were processed and normalized using the ExpressionFileCreator module implemented in GenePattern platform (http://www.broadinstitute.org/cancer/software/ genepattern/). The Robust Multichip Average normalized and $\log _{2}$-transformed expression values were used for downstream differential analysis. Significance Analysis of Microarrays in MeV TiGR Software was used to compute the fold change differences in genes comparing the controls to the diabetic mice. Significance was assessed at an FDR of $<0.1$. Downstream functional analysis of enriched pathways was generated using Ingenuity Pathway Analysis (QIAGEN). Raw and processed microarray data are available in the Gene Expression Omnibus repository (GSE86300 [kidney cortex] and GSE27382 [SCN]). 
Statistics. All MS data were $\log _{2}$ transformed prior to statistical analysis. Data analysis was performed using GraphPad Prism 6.0 (GraphPad Software). Box-and-whisker plots were used to present the data, with whiskers denoting the maximum and minimum values. Comparisons between groups were performed using unpaired 2-tailed Student's $t$ test, Welch's unequal variances $t$ test, 1-way ANOVA with Tukey's post hoc test for multiple comparisons, or Kruskal-Wallis test with Dunn's post-hoc test for multiple comparisons. Significance was defined as $P<0.05$.

Study approval. Animal experiments were approved by the University of Michigan Institutional Animal Care and Use Committee and performed in accordance with their policies. The institutional review board of the National Institute of Diabetes and Digestive and Kidney Diseases approved the Southwestern American Indians study. The Institutional review board of the Helsinki University Central Hospital, Finland, approved the FinnDiane study, and the FIND study was completed in accordance with the principles of the Declaration of Helsinki. For each study, written informed consent was obtained from all participants.

\section{Author contributions}

TWG, CFB, ELF, MK, FCB, and SP conceived and supervised the study. KMS, PK, JB, VN, LMH, JH, $\mathrm{HZ}, \mathrm{CL}$, and NRQ conducted the experiments. All authors analyzed the data and interpreted results. PHG, RGN, MD, KS, JR Schelling, and JR Sedor obtained and provided access to clinical samples. KMS, LMH, $\mathrm{ELF}, \mathrm{FCB}$, and SP wrote the manuscript. All authors reviewed and revised the manuscript.

\section{Acknowledgments}

Research reported in this publication was supported in part by grants from the National Institutes of Health: DK094292, DK089503, DK082841, DK081943, and DK097153 (SP), DK094352 (KS), DK089117 and DK106304 (SAS), and DK59997 and DK57329 (JR Sedor) from the National Institute of Diabetes and Digestive and Kidney Diseases; EY20582 (TWG and SFA) from the National Eye Institute; Michigan Institute of Clinical and Health Research CTSA Award 2UL1TR000433 (KMS) from the National Center for Advancing Translational Sciences; M01-RR-000080 from the Clinical Research Unit at Case Western Reserve University (JR Sedor); and from the Intramural Research Program of the National Institute of Diabetes and Digestive and Kidney Diseases (RGN). Additional support was provided by the American Diabetes Association, the Program for Neurology Research and Discovery, and Novo Nordisk Foundation NNF14SA0006 (ELF), the Central Society for Clinical and Translational Research (SAS), the Juvenile Diabetes Research Foundation (postdoctoral fellowship to LMH), and the Department of Medicine at the University of California, San Diego. The Finnish Diabetic Nephropathy Study was supported by grants from the Folkhälsan Research Foundation, the Wilhelm and Else Stockmann Foundation, the Academy of Finland, the Novo Nordisk Foundation, and by EVO governmental grant (TYH2011227). FCB, CFB, ELF, and TWG are supported by the Taubman Institute at the University of Michigan.

Address correspondence to: Subramaniam Pennathur, University of Michigan, 5309 Brehm Center, 1000 Wall Street, Ann Arbor, Michigan 48105, USA. Phone: 734.764.3269; E-mail: spennath@umich.edu.

1. Centers for Disease Control and Prevention. National diabetes fact sheet: national estimates and general information on diabetes and prediabetes in the United States, 2011. http://www.cdc.gov/diabetes/pubs/pdf/ndfs_2011.pdf. Accessed September 3, 2016.

2. Naresh Kumar R, Sundaram R, Shanthi P, Sachdanandam P. Protective role of 20-OH ecdysone on lipid profile and tissue fatty acid changes in streptozotocin induced diabetic rats. Eur J Pharmacol. 2013;698(1-3):489-498.

3. Sundaram R, Shanthi P, Sachdanandam P. Effect of iridoid glucoside on plasma lipid profile, tissue fatty acid changes, inflammatory cytokines, and GLUT4 expression in skeletal muscle of streptozotocin-induced diabetic rats. Mol Cell Biochem. 2013;380(1-2):43-55.

4. Vallon V. The proximal tubule in the pathophysiology of the diabetic kidney. Am J Physiol Regul Integr Comp Physiol. 2011;300(5):R1009-R1022.

5. Bonventre JV. Can we target tubular damage to prevent renal function decline in diabetes? Semin Nephrol. 2012;32(5):452-462.

6. Cohen JJ, Barac-Nieto M. Renal metabolism of substrates in relation to renal function. In: Orloff J, Berliner RW, eds. Handbook of Physiology Section 8: Renal Physiology. Washington, DC, USA: American Physiology Society; 1973:909-1001.

7. Meyer C, Nadkarni V, Stumvoll M, Gerich J. Human kidney free fatty acid and glucose uptake: evidence for a renal glucose-fatty acid cycle. Am J Physiol. 1997;273(3 Pt 1):E650-E654.

8. Wirthensohn G, Guder WG. Renal substrate metabolism. Physiol Rev. 1986;66(2):469-497.

9. Greene DA, Winegrad AI. In vitro studies of the substrates for energy production and the effects of insulin on glucose utilization in the neural components of peripheral nerve. Diabetes. 1979;28(10):878-887. 
10. Hinder LM, Vivekanandan-Giri A, McLean LL, Pennathur S, Feldman EL. Decreased glycolytic and tricarboxylic acid cycle intermediates coincide with peripheral nervous system oxidative stress in a murine model of type 2 diabetes. J Endocrinol. 2013;216(1):1-11

11. Ola MS, et al. Analysis of glucose metabolism in diabetic rat retinas. Am J Physiol Endocrinol Metab. 2006;290(6):E1057-E1067.

12. Ng SK, et al. Cancer-like metabolism of the mammalian retina. Clin Experiment Ophthalmol. 2015;43(4):367-376.

13. Atsuzawa $\mathrm{K}$, et al. Immunohistochemical localization of mitochondrial fatty acid $\beta$-oxidation enzymes in Müller cells of the retina. Histochem Cell Biol. 2010;134(6):565-579.

14. Sharma K, McCue P, Dunn SR. Diabetic kidney disease in the db/db mouse. Am J Physiol Renal Physiol. 2003;284(6):F1138-F1144.

15. Brosius FC, et al. Mouse models of diabetic nephropathy. J Am Soc Nephrol. 2009;20(12):2503-2512.

16. Koves TR, et al. Mitochondrial overload and incomplete fatty acid oxidation contribute to skeletal muscle insulin resistance. Cell Metab. 2008;7(1):45-56

17. Sullivan KA, et al. Mouse models of diabetic neuropathy. Neurobiol Dis. 2007;28(3):276-285.

18. Pande M, et al. Transcriptional profiling of diabetic neuropathy in the BKS db/db mouse: a model of type 2 diabetes. Diabetes. 2011;60(7):1981-1989.

19. Bogdanov $\mathrm{P}$, et al. The $\mathrm{db} / \mathrm{db}$ mouse: a useful model for the study of diabetic retinal neurodegeneration. PLoS One. 2014;9(5):e97302.

20. Passarella S, de Bari L, Valenti D, Pizzuto R, Paventi G, Atlante A. Mitochondria and L-lactate metabolism. FEBS Lett. 2008;582(25-26):3569-3576.

21. Wellen KE, Hatzivassiliou G, Sachdeva UM, Bui TV, Cross JR, Thompson CB. ATP-citrate lyase links cellular metabolism to histone acetylation. Science. 2009;324(5930):1076-1080.

22. Wang Q, et al. Acetylation of metabolic enzymes coordinates carbon source utilization and metabolic flux. Science. 2010;327(5968):1004-1007.

23. Zhao S, et al. Regulation of cellular metabolism by protein lysine acetylation. Science. 2010;327(5968):1000-1004

24. Li T, et al. Glyceraldehyde-3-phosphate dehydrogenase is activated by lysine 254 acetylation in response to glucose signal. $J$ Biol Chem. 2014;289(6):3775-3785

25. Beeson CC, Beeson GC, Schnellmann RG. A high-throughput respirometric assay for mitochondrial biogenesis and toxicity. Anal Biochem. 2010;404(1):75-81.

26. Breyer MD, et al. Diabetic nephropathy: a national dialogue. Clin J Am Soc Nephrol. 2013;8(9):1603-1605.

27. Fufaa GD, et al. Structural Predictors of Loss of Renal Function in American Indians with Type 2 Diabetes. Clin J Am Soc Nephrol. 2016;11(2):254-261.

28. Weil EJ, et al. Effect of losartan on prevention and progression of early diabetic nephropathy in American Indians with type 2 diabetes. Diabetes. 2013;62(9):3224-3231.

29. Tolonen N, et al. Relationship between lipid profiles and kidney function in patients with type 1 diabetes. Diabetologia. 2008;51(1):12-20.

30. Knowler WC, et al. The Family Investigation of Nephropathy and Diabetes (FIND): design and methods. J Diabetes Complicat. 2005;19(1):1-9.

31. Brownlee M. Biochemistry and molecular cell biology of diabetic complications. Nature. 2001;414(6865):813-820.

32. Nishikawa T, et al. Normalizing mitochondrial superoxide production blocks three pathways of hyperglycaemic damage. Nature. 2000;404(6779):787-790.

33. Du X, et al. Inhibition of GAPDH activity by poly(ADP-ribose) polymerase activates three major pathways of hyperglycemic damage in endothelial cells. J Clin Invest. 2003;112(7):1049-1057.

34. Giacco F, Brownlee M. Oxidative stress and diabetic complications. Circ Res. 2010;107(9):1058-1070.

35. Dugan LL, et al. AMPK dysregulation promotes diabetes-related reduction of superoxide and mitochondrial function. $J$ Clin Invest. 2013;123(11):4888-4899.

36. Randle PJ, Garland PB, Hales CN, Newsholme EA. The glucose fatty-acid cycle. Its role in insulin sensitivity and the metabolic disturbances of diabetes mellitus. Lancet. 1963;1(7285):785-789.

37. Shokolenko I, LeDoux S, Wilson G, Alexeyev M. Mitochondrial DNA damage, repair, degradation and experimental approaches to studying these phenomena. In: Storici F, ed. DNA Repair — On the Pathways to Fixing DNA Damage and Errors. InTech; 2011. http://www.intechopen.com/books/dna-repair-on-the-pathways-to-fixing-dna-damage-and-errors/mitochondrial-dna-damage-repair-degradation-and-experimental-approaches-to-studying-these-phenomena. Published September 9, 2011. Accessed August 18, 2016.

38. Friederich M, Fasching A, Hansell P, Nordquist L, Palm F. Diabetes-induced up-regulation of uncoupling protein-2 results in increased mitochondrial uncoupling in kidney proximal tubular cells. Biochim Biophys Acta. 2008;1777(7-8):935-940

39. Peti-Peterdi J. High glucose and renin release: the role of succinate and GPR91. Kidney Int. 2010;78(12):1214-1217.

40. Toma I, et al. Succinate receptor GPR91 provides a direct link between high glucose levels and renin release in murine and rabbit kidney. J Clin Invest. 2008;118(7):2526-2534.

41. He W, et al. Citric acid cycle intermediates as ligands for orphan G-protein-coupled receptors. Nature. 2004;429(6988):188-193.

42. de Cavanagh EM, Inserra F, Ferder M, Ferder L. From mitochondria to disease: role of the renin-angiotensin system. Am J Nephrol. 2007;27(6):545-553.

43. Lee DY, et al. Nox4 NADPH oxidase mediates peroxynitrite-dependent uncoupling of endothelial nitric-oxide synthase and fibronectin expression in response to angiotensin II: role of mitochondrial reactive oxygen species. J Biol Chem. 2013;288(40):28668-28686.

44. You YH, Quach T, Saito R, Pham J, Sharma K. Metabolomics Reveals a Key Role for Fumarate in Mediating the Effects of NADPH Oxidase 4 in Diabetic Kidney Disease. J Am Soc Nephrol. 2016;27(2):466-481.

45. Kosanam H, et al. Diabetes induces lysine acetylation of intermediary metabolism enzymes in the kidney. Diabetes. 2014;63(7):2432-2439.

46. Reddy MA, Zhang E, Natarajan R. Epigenetic mechanisms in diabetic complications and metabolic memory. Diabetologia. 2015;58(3):443-455. 
47. Zhang X, Tang N, Hadden TJ, Rishi AK. Akt, FoxO and regulation of apoptosis. Biochim Biophys Acta. 2011;1813(11):1978-1986.

48. Banks AS, et al. Dissociation of the glucose and lipid regulatory functions of FoxO1 by targeted knockin of acetylation-defective alleles in mice. Cell Metab. 2011;14(5):587-597.

49. Kato M, et al. Role of the Akt/FoxO3a pathway in TGF-beta1-mediated mesangial cell dysfunction: a novel mechanism related to diabetic kidney disease. J Am Soc Nephrol. 2006;17(12):3325-3335.

50. Olmos Y, et al. Mutual dependence of Foxo3a and PGC-1alpha in the induction of oxidative stress genes. J Biol Chem. 2009;284(21):14476-14484.z

51. Kubli DA, Gustafsson ÅB. Unbreak my heart: targeting mitochondrial autophagy in diabetic cardiomyopathy. Antioxid Redox Signal. 2015;22(17):1527-1544.

52. Mammucari C, et al. FoxO3 controls autophagy in skeletal muscle in vivo. Cell Metab. 2007;6(6):458-471.

53. Kume S, et al. Calorie restriction enhances cell adaptation to hypoxia through Sirt1-dependent mitochondrial autophagy in mouse aged kidney. J Clin Invest. 2010;120(4):1043-1055.

54. Joo KW, et al. Dipeptidyl peptidase IV inhibitor attenuates kidney injury in rat remnant kidney. BMC Nephrol. 2013;14:98.

55. Chung HW, et al. High-fat diet-induced renal cell apoptosis and oxidative stress in spontaneously hypertensive rat are ameliorated by fenofibrate through the PPAR $\alpha$-FoxO3a-PGC-1 $\alpha$ pathway. Nephrol Dial Transplant. 2012;27(6):2213-2225

56. Das F, et al. High glucose forces a positive feedback loop connecting Akt kinase and FoxO1 transcription factor to activate mTORC1 kinase for mesangial cell hypertrophy and matrix protein expression. J Biol Chem. 2014;289(47):32703-32716

57. Higgins GC, Coughlan MT. Mitochondrial dysfunction and mitophagy: the beginning and end to diabetic nephropathy? Br $J$ Pharmacol. 2014;171(8):1917-1942.

58. Wrighton KH. Metabolism: putting energy into mitophagy. Nat Rev Mol Cell Biol. 2013;14(6):324

59. Melser S, et al. Rheb regulates mitophagy induced by mitochondrial energetic status. Cell Metab. 2013;17(5):719-730.

60. Sharma K, et al. Metabolomics reveals signature of mitochondrial dysfunction in diabetic kidney disease. J Am Soc Nephrol. 2013;24(11):1901-1912.

61. Kang HM, et al. Defective fatty acid oxidation in renal tubular epithelial cells has a key role in kidney fibrosis development. Nat Med. 2015;21(1):37-46.

62. Herman-Edelstein M, Scherzer P, Tobar A, Levi M, Gafter U. Altered renal lipid metabolism and renal lipid accumulation in human diabetic nephropathy. J Lipid Res. 2014;55(3):561-572.

63. Li M, et al. GC/TOFMS analysis of metabolites in serum and urine reveals metabolic perturbation of TCA cycle in $\mathrm{db} / \mathrm{db}$ mice involved in diabetic nephropathy. Am J Physiol Renal Physiol. 2013;304(11):F1317-F1324.

64. Myers BD, et al. Progression of overt nephropathy in non-insulin-dependent diabetes. Kidney Int. 1995;47(6):1781-1789.

65. Schmid H, et al. Modular activation of nuclear factor-kappaB transcriptional programs in human diabetic nephropathy. Diabetes 2006;55(11):2993-3003.

66. Cohen CD, Frach K, Schlöndorff D, Kretzler M. Quantitative gene expression analysis in renal biopsies: a novel protocol for a high-throughput multicenter application. Kidney Int. 2002;61(1):133-140.

67. Mäkinen VP, et al. Diagnosing diabetic nephropathy by 1H NMR metabonomics of serum. MAGMA. 2006;19(6):281-296. 